\title{
2007/37
}

\section{A theory of dynamics and inequalities under epidemics}

Raouf Boucekkine and Jean-Pierre Laffargue 


\title{
CORE DISCUSSION PAPER
} $2007 / 37$

\section{A theory of dynamics and inequalities under epidemics}

\author{
Raouf BOUCEKKINE ${ }^{1}$ and Jean-Pierre LAFFARGUE ${ }^{2}$
}

June 2007

\begin{abstract}
We develop a tractable general theory for the study of the economic and demographic impact of epidemics. In particular, we analytically characterize the short and medium term consequences of epidemics for population size, age pyramid, economic performance and income distribution. To this end, we develop a three-period overlapping generations where altruistic parents choose optimal health expenditures for their children and themselves. The survival probability of (junior) adults and children depend on such investments. Agents can be skilled or unskilled. The model emphasizes the role of orphans. Orphans are not only penalized in front of death, they are also penalized in the access to education. Epidemics are modeled as one period exogenous shocks to the survival rates. We identify three kinds of epidemics depending on how the epidemic shock alters the marginal efficiency of health expenditures. We first study the demographic dynamics, and prove that while a one-period epidemic shock has no permanent effect on income distribution, it can perfectly alter it in the short and medium run. We then study the impact of the three kinds of epidemics when they hit children and/or junior adults. We prove that while the three epidemics have significantly different demographic implications in the medium run, they all imply a worsening in the short and medium run of economic performance and income distribution. In particular, the distributional implications of the model mainly rely on orphans: if orphans are more penalized in the access to a high level of education than in front of death, they will necessarily lead to the medium-term increase in the proportion of the unskilled, triggering the impoverishment of the economy at that time horizon.
\end{abstract}

Keywords: epidemics, orphans, income distribution, endogenous survival, medium-term dynamics

JEL classification: O1, D9, I1, I2

${ }^{1}$ Department of Economics and CORE, Université catholique de Louvain, Belgium. E-mail: boucekkine@core.ucl.ac.be 2 University Paris I, PSE and CEPREMAP, Paris, France. E-mail: laffargue@pse.ens.fr

This paper has benefited from several suggestions and comments by Bruno Decreuse, David de la Croix, Mathias Doepke, Cecilia Garcia-Penalosa, Alan Kirman, John Knowles, Omar Licandro, Omer Moav, Rodrigo Suarez, Uwe Sunde, Alain Trannoy, and participants in meetings and seminars held at IZA-Bonn, GREQAM-Marseille, the Technical University of Vienna, and Université catholique de Louvain. Boucekkine acknowledges the financial support of the Belgian research programmes PAI P5/10 and ARC 03/08-302. The usual disclaimer applies.

This paper presents research results of the Belgian Program on Interuniversity Poles of Attraction initiated by the Belgian State, Prime Minister's Office, Science Policy Programming. The scientific responsibility is assumed by the authors. 


\section{Introduction}

Though the study of the economic effects of epidemics has always been of interest to many economists (see for example Hirshleifer, 1987), the more recent HIV/AIDS pandemic and its apparent massive demographic effects, especially in sub-Saharan Africa, has suggested an exceptionally abundant literature. Yet, there is no common view of the medium and long run consequences of such an epidemic on economic growth so far.

In particular, the impact of AIDS on economic growth has been investigated in many empirical studies ${ }^{4}$. While Bloom and Mahal (1997) found a statistically insignificant coefficient on the AIDS variable and conclude that AIDS has had little impact on growth, McDonald and Roberts (2006) identified a strong effect of the stock of general health on average income in developing countries, and concluded that the marginal impact on income per capita of a $1 \%$ increase in HIV prevalence rate is minus $0.59 \%$ in Africa. Of course, the discrepancy can be partially attributed to differences in the experimental framework. It can be also attributed to the period of analysis, 1980-1992 in the case of Bloom and Mahal, and 1984-1999 in MacDonald and Roberts. Unfortunately, the discrepancy is still noticeable in the most recent wave of contributions to this field (see Young, 2005, Vs Kalemli-Ozcan, 2006).

Such a disagreement on the growth effects of AIDS is also apparent in the empirical literature which studies the impact of the Spanish flu (see the excellent work of Brainerd and Siegler, 2003). At the theoretical level, the discrepancy in the evaluation of the effects of an epidemic on economic growth is especially neat in the benchmark growth models, as clearly reflected in the analysis of Boucekkine, Diene and Azomahou (2007). Consider for example the celebrated textbook by Barro and Sala-I-Martin (1995), chapter 5. Two models are considered in this chapter. Both use two production factors: physical capital and human capital. The economy is on a reference balanced growth path when an epidemic, which takes place at date 0 , destroys a part of the human capital, but leaves physical capital intact. In the first model, the sector producing the human capital uses the same technology as the sector producing consumption goods and physical capital; it is therefore a one-sector model. However, investments in both factors must be non-negative (irreversibility). Then the epidemic creates an imbalance between the two factors. The economy reacts by setting the investment in physical capital to zero, but also by reducing households' consumption. The correction of the

\footnotetext{
${ }^{4}$ For an excellent review see United Nations (2004).
} 
imbalance and the reduction in consumption increase the growth rate of the production of the physical good above its reference value. Of course, this growth rate will decline monotonically over time until it reaches its initial value.

The second case considered by Barro and Sala-i-Martin is the celebrated Lucas-Uzawa model (see for example Lucas, 1988). Education, the sector producing human capital, only uses this factor as input. The production of consumption good and physical capital uses both factors. Then, an epidemic increases the scarcity of human capital, and the wage rate. The high cost of operation for the education sector will motivate people to allocate human capital to production of goods, rather than to education, the sector that produces the relatively scarce factor. This effect tends to retard the economy's growth rate. The growth rate of gross output (including the production of new human capital) will decrease at the time of the epidemic, then it will increase monotonically over time until it reaches its reference value.

Hence, the predictions of the two-sector model for economic growth are exactly the opposite of those of the one-sector prototype ${ }^{5}$. There are obvious limitations in the analysis of the macroeconomic impact of epidemics as performed in textbook theory. In particular, all the models listed above consider that human capital, which aggregates the education level of the population and sometimes its health status, is similar to physical capital. However, human capital (education and health) is embodied in individuals, inducing possible big differences concerning the mechanisms of investment in physical capital. For instance, the death of a child or an elderly has no effect on the level of the human capital used in production. Its economic effects will be very different from the death of workers in their twenties or thirties, which brings the destruction of human capital progressively brought up in them through child rearing, formal education and learning on the job.

Another specificity of the human capital (education and health) is that the amount of it embodied in a person strongly results from decisions taken by his parents. Bowles and Gentis (2002) quote a series of empirical results for the United States. A son born in the highest income decile has a probability of $22.9 \%$ to reach the same decile and a probability of $2.4 \%$ to reach the lowest income decile. A son born in the lowest income decile has a probability of $1.3 \%$ to reach the highest decile and a probability of $31.2 \%$ to reach the lowest decile. Grawe

\footnotetext{
${ }^{5}$ Incidentally, the latter delivers the same prediction as the even more standard Solow model. In such a model, the initial effect of an epidemic is to increase the amount of capital per worker and output per worker. After the initial shock, the economy will gradually converge back to its steady-state, and the growth rate of output per worker will be less than its steady state value during this transition.
} 
and Mulligan (2002) review cross-country evidence showing that countries with lower public provision of human capital experience smaller intergenerational mobility. For instance, less developed countries exhibit strong intergenerational transmission. The connection between the absence of intergenerational mobility and education is well documented. Bowles and Gentis show that this situation can also be linked to the health of children, which is itself a function of their parents' income (see also Case, Lubotsky and Paxson, 2001).

In this paper, we develop a theory of epidemics taking into account the embodied nature of human capital, and enhancing the role of parental decisions. For the generality of the theory, we consider both child and adult mortality: since human capital is embodied in individuals and since parents decide about the education and health expenditures of their children, the economic and demographic impacts of epidemics is likely to tightly depend on the age-profile of the induced mortality. To increase the scope of our theory, we shall also allow for any profile of the marginal efficiency of health expenditures under epidemics. Define at the minute the marginal efficiency of health expenditures is the impact on the survival probability of an individual of a marginal increase in the latter expenditures. We will allow for different epidemiological situations in which this marginal efficiency is either increased, decreased or unchanged.

A last characteristic of our general framework is to explicitly account for the role of orphans. When young adults die, not only do they reduce the amount of labour and human capital used in production, but they also leave orphans behind them. To show how this effect can be disastrous, we can quote the following extract of an article published by The Economist (2003) " ... one-in-ten sub-Saharan children is now an orphan. A third of these are the result of AIDS. Orphaning rates above 5\% worry UNICEF because they exceed the capacity of local communities to care for parentless children. So do places such as Zambia, where almost $12 \%$ of children are AIDS orphans.... Orphans tend to be poorer than non orphans, and to face a higher risk of malnutrition, stunting and death - even if they are free of HIV themselves. Orphans are less likely to attend school because they cannot afford the fees but also because step-parents tend to educate their own children first". Case, Pakson and Ableidinger (2004) give interesting complements to this view. Orphans live in foster families who discriminate against them and in favour of the children of the family head. The probability of the school enrolment of an orphan is inversely proportional to the degree of relatedness of the child to the household head. Gertler, Levine and Martinez (2003) show that parental loss does not operate 
only through a reduction in household resources. Parental presence, including the loss of mentoring, the transmission of values and emotional and psychological support, plays an important role in investment in child human capital.

There are several papers developing computable general equilibrium models to investigate the effects of AIDS, and giving an important role to the increase in the number of orphans. For example, Bell, Devarajan and Gersbach (2003) develop such a model applied to the South African case. The authors emphasise the formation of human capital and transmission mechanism across generations and conclude to a very negative effect of the epidemic on longrun growth, with a large proportion of families and their offspring falling in a poverty trap. So, a transitory shock can have permanent effects. A similar model was developed by Corrigan, Glomm and Mendez (2004), who also conclude that the growth effects of AIDS are large. In their paper, children receive a different level of education if their parents are healthy or ill. However, when they grow up and become adult, the available human capital of their cohort is reallocated in an egalitarian way between all its members. This redistribution, which forsakes the assumption of embodiment of the investment in education, simplifies the simulation of the model, but contradicts the optimisation program of the parents that does not anticipate it. In the paper by Bell, Devarajan and Gersbach children are ranked by increasing human capital then divided into a finite number of classes. The reallocation of the human capital occurs inside each of these classes. Thus, these authors approximate a continuous distribution by a discrete distribution. As this approximation can be as precise as desired, this solution is more acceptable than the previous one. Finally, none of these papers discusses the effect of an epidemic on the marginal efficiency of health expenditures.

Our paper takes a broader perspective by considering any age-profile of mortality and any marginal efficiency of medication under epidemics. The demographic and economic properties of the model are fully analytically investigated in the short, medium and long-run, which is already a contribution to the literature. In our model, people live for three periods, successively as children, junior adults and senior adults. A junior adult has an exogenous number of children and is perfectly altruistic that is he only cares for the survival of his children and the social position they will get. He invests in his own health and education, and in the health and education of his children ${ }^{6}$. The probability for a child to reach a high level of human capital is

\footnotetext{
6 Ricci and Zachariadis (2006) find that investment in education and in health are positively related at equilibrium and have a reinforced impact on longevity. Cutler, Deaton and Lleras-Muney (2005) show that the
} 
independent of the levels of the human capital of his parent, under the conditions that he survives and that his parent survives and is able to bring him up. Thus, we have eliminated the traditional channel of the cultural heritage to focus on alternative channels which work through education and health and investments in both. The probabilities of survival of a child and of a junior adult depend on the amounts of money spent by the junior adult for his own human capital and for the one of his children.

The credit market is incomplete: parents cannot finance spending on their children by borrowing against their higher expected income, which will result from this spending. So, health and education spending and the probabilities of survival will be low if parents are poor. Moreover, if a parent dies and if his children become orphans, their probabilities of survival will be lowered. Finally, we will assume that an orphan has a lower probability to reach a high level of human capital than a child brought up by living parents. Accordingly, a key feature of the paper is to consider a crucial dimension of inequality, namely inequality in front of death. Inequality between children has several causes. First, the children of less educated parents who have survived and who bring them up have a higher probability of dying before growing adults because their parents spend less on their health and education. Secondly, less educated parents spend less on their own education and health and have a higher probability to die and to be unable to bring their children up.

A key contribution of our paper is the analytical study of the impact of epidemics on the income distribution in the short, medium and long-run. It should be noted that the papers focusing on AIDS usually comment on the changes in the distributions of human capital and income possibly following the epidemic although they do not fully investigate them. The only theoretical paper we know, which investigates the links between health spending, mortality and the persistence of inequality across generations, is by Chakraborty and Das (2005). These authors base their analysis of the persistence of poverty on the fact that poor parents invest less in their own health and so have a high probability of dying. Thus, they save little and leave to their children a small bequest if they survive and a still smaller bequest if they die. The paper assumes that parents only care for the health of their children if they are themselves alive when their children grow. However, parents cannot buy annuities against the saving they will leave in the case of their premature death (so, in this situation, children get an unplanned

application of scientific advance and technical progress, which is facilitated by education, explains a great deal of the inequality in health inside a country. 
bequest). An extension of the paper introduces the possibility of investing, not only in the health of parents, but in the education of children too. The productivity of labour depends on both these investments. Nonetheless, these authors do not consider investments in the health of children nor their survival probability.

The paper is organised as follows. The second section presents the model and its short run equilibrium. The third section is devoted to the transitory dynamics and the long run equilibrium of demographic variables. The fourth section investigates the economic and demographic effects of epidemics. The fifth section concludes on the possible role of fertility behaviour response to epidemics.

\section{The model: behaviour of the agents and temporary equilibrium}

We consider a discrete time, perfect foresight dynamic model of a small open economy. People live for three periods, successively as children, junior adults and senior adults. We will start by examining the choices of a junior adult in an given period denoted $t$. In a second paragraph we will describe the temporary equilibrium of the model in this period. To ease the exposition and to be able to bring out a fully analytical characterization, we shall refer to a single good, health care. The latter should be taken in the much broader sense of any investment raising human capital (including education).

\subsection{The choices of a junior adult}

A junior adult enters period $t$ with an endowment in human capital $h$. Healthcare is the only good existing in the economy. It is produced by firms, which use human capital as their unique input and which operate under constant returns. We will assume that the productivity of human capital is equal to 1 and that firms make no profit. Thus, $h$ can also be interpreted as the earnings of the agent. The agent sets his saving $s$ and his investment in health $l$ for the period, under the budget constraint

$$
\text { (1) } h=s+l
$$

Spending on health has an effect on the lifetime of the agent. His probability of being alive in period $t+1$ (as a senior adult) is $\pi(l)$. At the end of period $t$ the agent will have an exogenous number $n$ of children. Senior adults receive no wages. This assumption will simplify the model in directions that we are not very interested to investigate. The agent will 
invest $e_{+1}$ in the health of each of his children. The probability for each of them to be alive at the beginning of period $t+2$ will depend on this investment. If the agent is alive in period $t+1$ and can take care of his children, this probability will be $\lambda\left(e_{+1}\right)$. If he is dead and if his children are orphans, this probability will be $c \lambda\left(e_{+1}\right)$, with $0<\underline{c} \leq c<1$. The saving of the agent in period $t, s$, is lent on the international capital market at the exogenous and constant capitalisation rate $R>1$. The budget constraint of the agent in period $t+1$ is:

$$
\text { (2) } R s=n e_{+1}
$$

We notice that the amount invested by the agent in the health of his children will be the same if the agent dies or stays alive at the end of period $t$. This investment is equal to the capitalisation of the saving made in period $t$. The intertemporal budget constraint of the agent is

$$
\text { (3) } R h=l R+n e_{+1}
$$

To simplify the model we will assume that human capital can take only two values: $h^{-}$and $h^{+}$, with: $0<h^{-}<h^{+}$. We will assume that a child who has living parents and who stays alive has a probability $p$ of obtaining a human capital of $h^{+}$and a probability $1-p$ of obtaining a human capital of $h^{-}$. An orphan who stays alive has the probability $q$ of obtaining the high level of human capital and $1-q$ of obtaining the low level of human capital. We assume that $0 \leq q<p \leq 1$.

Our junior adult has the following utility function in period $t$

$$
\text { (4) } U \equiv n \lambda\left(e_{+1}\right)\left\{\pi(l) v\left[p\left(h^{+}-h^{-}\right)+h^{-}\right]+[1-\pi(l)] v c\left[q\left(h^{+}-h^{-}\right)+h^{-}\right]\right\}
$$

The junior adult is wholly altruistic. His utility only depends on the expected human capital accumulated by his children who will reach the adult age. Our specification is in the spirit of evolutionary biology (see Galor and Moav, 2002 and 2005, for an earlier attempt to account for evolutionary biology ingredients in the theory of economic growth). Consistently with the traditional Darwinian theory, the parent should maximize the probability of survival and quality of her children. Nonetheless, in contrast to Galor and Moav (2005), we keep the number of offspring exogenously fixed. Since we are primarily interested in the impact of epidemics and since it is not clear so far whether the latter has lead and/or will lead to a substantial change in optimal fertility behaviour, we have preferred to develop our benchmark theory with fertility fixed. On the other hand, adding endogenous fertility to the model would require additional adjustments which will reduce sharply its tractability. As it will be clear 
along the way, the model is already heavy to handle algebraically. In the concluding section of the paper, we come back to the current AIDS related literature on fertility and examine the consequences of an exogenous fertility shock.

If the junior adult reaches the age of senior adult, he will bring his children up, which will increase their probability of survival and their expected levels of human capital. $v h^{+}\left(v h^{-}\right)$ represents the satisfaction a child brings to his parent when he reaches the adult age with the level of human capital $h^{+}\left(h^{-}\right)$. We assume that $v>0$. When the child dies this satisfaction is 0 . We will introduce the following notations

$$
\text { (5) } r_{1}=v\left[p\left(h^{+}-h^{-}\right)+h^{-}\right], r_{2}=v c\left[q\left(h^{+}-h^{-}\right)+h^{-}\right] \text {and } r=r_{1} / r_{2}-1 \text {. }
$$

The utility function of our junior adult in period $t$ becomes, after having removed a constant multiplicative term, $U \equiv \lambda\left(e_{+1}\right)[\pi(l) r+1] . r$ represents the premium in satisfaction brought by children, when their parent stays alive, or if one prefers, the utility for parents of staying alive. In this case, the probability of survival of each child is higher (by a factor $1 / c$ ) and his expected level of human capital is higher too. $r$, is an increasing function of the inequality in earnings, $\left(h^{+}-h^{-}\right) / h^{-}$, which is expected for the next period. In the following exercises of comparative static, we will assume that $h$ and $r$ can change independently. Finally, our junior adult must solve in period $t$ the program

$$
\text { (6) } \begin{gathered}
\operatorname{Max}_{l, e_{+1}} \lambda\left(e_{+1}\right)[\pi(l) r+1] \\
R h=l R+n e_{+1} \\
l, e_{+1} \geq 0
\end{gathered}
$$

Before solving this program we must give precise specifications of the survival functions:

(7) $\lambda\left(e_{+1}\right)=\left(A e_{+1}+A^{\prime}\right)^{1-\alpha} /(1-\alpha)$, if $0 \leq A e_{+1}+A^{\prime} \leq(1-\alpha)^{1 /(1-\alpha)}$

$$
\lambda\left(e_{+1}\right)=1, \text { if } A e_{+1}+A^{\prime} \geq(1-\alpha)^{1 /(1-\alpha)}
$$

(8) $\pi(l)=\left(B l+B^{\prime}\right)^{1-\beta} /(1-\beta)$, if $0 \leq B l+B^{\prime} \leq(1-\beta)^{1 /(1-\beta)}$

$\pi(l)=1$, if $B l+B^{\prime} \geq(1-\beta)^{1 /(1-\beta)}$

with: $0<\beta, \alpha<1, A, B, B^{\prime}>0,0 \leq A^{\prime}<(1-\alpha)^{1 /(1-\alpha)}, B^{\prime}<(1-\beta)^{1 /(1-\beta)}$.

In the rest of the paper we will assume that we are always inside the intervals where both functions are strictly increasing. Deaton (2003) notices that health spending, the health state and the longevity of an individual are increasing and concave functions of his income: for instance the probability for somebody of dying between the ages of 50 and 60 is a decreasing 
convex function of his income. This concavity is a possible explanation of the impact of inequality on the average health state in a country, and it implies that some redistribution of income can increase average health.

Later on, we will define an epidemic as an (anticipated or unanticipated) decrease in one of the parameters of the survival function, lasting for one period. There are different kinds of epidemics hitting the generation of the junior adults. Indeed, an epidemic can result in the decrease of parameter $B$ or $B^{\prime}$ or both. We define the efficiency of adults' health spending as the derivative of their probability of survival with respect to health spending $\partial \pi(l) / \partial l=B\left(B l+B^{\prime}\right)^{-\beta}$.

We have:

$$
\begin{gathered}
\partial^{2} \pi(l) /(\partial l \partial B)=\left[(1-\beta) B l+B^{\prime}\right]\left(B l+B^{\prime}\right)^{-1-\beta}>0, \partial^{2} \pi(l) /\left(\partial l \partial B^{\prime}\right)=-\beta B\left(B l+B^{\prime}\right)^{-1-\beta}<0, \\
\text { and } \partial^{2} \pi(l) / \partial l^{2}=-\beta B^{2}\left(B l+B^{\prime}\right)^{-1-\beta}<0 .
\end{gathered}
$$

The efficiency of health spending decreases with an epidemic lowering $B$ and increases with an epidemic decreasing $B^{\prime}$. This efficiency decreases for a composite epidemic, which decreases the values of parameters $B$ and $B^{\prime}$ by the same proportion. Finally, the efficiency of health spending decreases with the amount of money spent on health, which is a reasonable result. Hereafter, we shall call an epidemic of a first kind a shock lowering $B$ and $B^{\prime}$ by the same magnitude. A shock decreasing $B^{\prime}$ (Resp. B) will be called an epidemic of the second (Resp. third) kind. The same considerations could be made on shocks affecting infant mortality, we shall therefore adopt the same terminology for epidemics lowering parameters A and A'.

With the survival functions given above, program (6) becomes

$$
\begin{aligned}
& \text { (9) } \underset{l, e_{+1}}{\operatorname{Max}}\left(A e_{+1}+A^{\prime}\right)^{1-\alpha}\left\lfloor r\left(B l+B^{\prime}\right)^{1-\beta} /(1-\beta)+1\right] /(1-\alpha) \\
& R h=l R+n e_{+1} \\
& l, e_{+1} \geq 0, \quad A e_{+1}+A^{\prime} \leq(1-\alpha)^{1 /(1-\alpha)}, B l+B^{\prime} \leq(1-\beta)^{1 /(1-\beta)}
\end{aligned}
$$

We make the following assumptions.

Assumption 1. The parameters of the model must satisfy the constraints

(10) $B h^{+}+\frac{n B}{R} \frac{A^{\prime}}{A}+B^{\prime} \leq(1-\beta)^{1 /(1-\beta)}\left[1+\frac{1-\alpha}{1-\beta}(1+1 / r)\right]$ 
(11) $B h^{+}+\frac{n B}{R} \frac{A^{\prime}}{A} \leq \frac{n B}{R} \frac{(1-\alpha)^{1 /(1-\alpha)}}{A}$

(12) $\frac{1-\alpha}{1-\beta} B^{\prime}+\frac{1-\alpha}{r} B^{\prime \beta}<\frac{n B}{R} \frac{A^{\prime}}{A}+B h^{-}$

(13) $\frac{n B}{R} \frac{A^{\prime}}{A}<\frac{1-\alpha}{r}\left(B h^{-}+B^{\prime}\right)^{\beta}$

Now, we can establish the following lemmas.

Lemma 1. Program (9) has a unique solution defined by the two equations

(14) $\frac{n B A^{\prime} /(A R)+B^{\prime}+B h}{B l+B^{\prime}}-\frac{1-\alpha}{r\left(B l+B^{\prime}\right)^{1-\beta}}=1+\frac{1-\alpha}{1-\beta}$

(15) $e_{+1}=\frac{R}{n}(h-l)$

Proof. Equation (15) is the constraint in program (9). We use this constraint to eliminate $e_{+1}$ from the objective function. This function is concave in $l$. Equation (14) is the first order conditions of the so-transformed objective function. Let us define the function $y(l) \equiv \frac{n B A^{\prime} /(A R)+B^{\prime}+B h}{B l+B^{\prime}}-\frac{1-\alpha}{r\left(B l+B^{\prime}\right)^{1-\beta}}$.

$y(0)=1+\frac{n B A^{\prime} /(A R)+B h}{B^{\prime}}-\frac{1-\alpha}{r B^{1-\beta}}>1+\frac{1-\alpha}{1-\beta}$, because of inequality (12)

$y(h)=1+\frac{n B A^{\prime} /(A R)}{B h+B^{\prime}}-\frac{1-\alpha}{r\left(B h+B^{\prime}\right)^{1-\beta}}<1<1+\frac{1-\alpha}{1-\beta}$, because of inequality (13)

$y(+\infty)=-0 . \quad y(l)$ has a unique minimum, which is negative, for $\left(B l+B^{\prime}\right)^{\beta}=\frac{r\left[n B A^{\prime} /(A R)+B^{\prime}+B h\right]}{(1-\alpha)(1-\beta)} . y(l)=0$ for $\left(B l+B^{\prime}\right)^{\beta}=\frac{r\left[n B A^{\prime} /(A R)+B^{\prime}+B h\right]}{(1-\alpha)}$. Thus, equation (12) defines a unique value for $l$, which is positive and smaller than $h$.

We have to check that this solution satisfies $B l+B^{\prime} \leq(1-\beta)^{1 /(1-\beta)}$. This is equivalent to $\left.y \mid(1-\beta)^{1 /(1-\beta)} / B-B^{\prime}\right] \leq 1+(1-\alpha) /(1-\beta)$, which results from inequality (10).

We also have to check that $A e_{+1}+A^{\prime}=A \frac{R}{n}(h-l)+A^{\prime} \leq(1-\alpha)^{1 /(1-\alpha)} \quad$ or $l \geq h+\frac{n}{A R}\left[A^{\prime}-(1-\alpha)^{1 /(1-\alpha)}\right]$. This condition is satisfied because of inequality (11). 
The two following lemmas describe in detail the characteristics of the optimal decisions taken by a junior adult, first concerning investment in his own health, then concerning investment in the health of his offspring.

Lemma 2. a) A well-endowed junior adult invests more in his health than a poorly endowed junior adult. b) The investment of a junior adult in his own health increases with his earnings and when there is an increase in the utility for parents of being alive. $c$ ) The investment of a junior adult in his own health decreases when the scale parameter B decreases (epidemic of the third kind) and increases when parameter B' decreases (epidemic of the second kind). This investment decreases if $B$ and $B^{\prime}$ decrease by the same percentage (epidemic of the first kind). d) The investment of a junior adult in his own health increases when the scale parameter of the survival function of his children A decreases (epidemic of the third kind), and decreases when parameter $A^{\prime}$ decreases (epidemic of the second kind). This investment does not change when parameters $A$ and $A^{\prime}$ decrease by the same percentage (epidemic of the first kind). f) Unless $A^{\prime}=0$, the investment of a junior adult in his own health depends on the number of his children: It increases when the later number goes up.

Lemma 3. a) A well-endowed junior adult invests more in the health of his children than a poorly endowed junior adult. $b$ ) The investment of a junior adult in the health of his children increases with his earnings and decreases when there is an increase in the utility for parents of being alive. $c$ ) The investment of a junior adult in the health of his children increases when the scale parameter $B$ decreases and decreases when parameter $B^{\prime}$ decreases. This investment increases if parameters $B$ and $B^{\prime}$ decrease by the same percentage. d) The investment of a junior adult in the health of his children decreases when the scale parameter of the survival function of his children $A$ decreases, and increases when parameter $A^{\prime}$ decreases. This investment stays unchanged if parameters $A$ and $A^{\prime}$ decrease by the same percentage. f) Unless $A^{\prime}=0$, the total investment of a junior adult in the health of his children decreases with the number of children.

The model has several worth-mentioning properties. First, and as announced in the introduction section, our model entails inequality in front of death. Children of parents with a low human capital have a higher probability of dying before growing. Moreover, such parents tend to spend less in their own health care (and education), and hence face a lower survival probability with the subsequent negative effect on the human capital of the resulting orphans. 
Second, the investment decisions taken by the junior adults are sensitive to exogenous changes in their survival function (Property c of Lemma 2 and 3) and to shifts in the survival function of their children (Property d of Lemma 2 and 3). Put in other words, an epidemic hitting young adults as an epidemic hitting their own children will have an impact on the investment decisions of these individuals.

The consequences of varying the life expectancy are extensively studied in the literature. Our model has some interesting predictions regarding this issue. In the standard theory relying on Blanchard-Yaari structures, life expectancy (or the mortality rate) is exogenous. A downward shift in the life expectancy generally decreases the marginal return to investment in this framework, implying less investment either in physical capital (as in the standard Blanchard model, 1985) and/or human capital (as in Boucekkine, de la Croix and Licandro, 2002). In our model, different kinds of epidemics can hit a generation of junior adults. The first and third kinds decrease the efficiency of adults' health investment and the second kind increases the efficiency of this investment. Moreover, life expectancy is no longer exogenous. When an epidemic of the first or third kind shortens the life expectancy of junior adults, the adults' health expenditure decreases for reasons similar to the ones we just gave and life expectancy decreases by more than what results from the direct effect of the epidemic. However, with an epidemic of the second kind, adults' health expenditure increases, which reduces the direct effects of the epidemic. The effects of these epidemics on children's heath investment are exactly opposite to those on their parents' health spending: it increases with the first kind of epidemic and decreases with the second kind.

Actually, our set-up has more subtle predictions concerning children's health care: the investment decisions of the parents are also sensitive to an anticipated drop in the life expectancy of their children. If this drop decreases the efficiency of children's health investment, then, their parents will spend more on their own health and less on their children's health. If this drop increases the efficiency of children's health investment, then their parents will invest less in their own health and more on the health of their children. The second result is easy to accept. Parents increase their health expenditures in the benefit of their children when they are subject to an exogenous deterioration of their health precisely because their utility is entirely determined by the expected human capital accumulated by children who will reach the adult age. To understand the first result we must remember that children benefit from growing under living parents: orphans will have a higher probability of dying and of 
reaching a high level of human capital. Thus, parents who decrease the investment in the health of their children when their probability of survival has been decreased, continue of being perfectly altruistic. They only know that investing in their own health instead of the health of their children is the most efficient way of improving the welfare of their children.

We showed that $\partial^{2} \pi(l) /(\partial l \partial B)>0$ and $\partial^{2} \pi(l) /\left(\partial l \partial B^{\prime}\right)<0$. As health spending is higher for a well-endowed than for a poorly endowed junior adult we get the following result. An epidemic of the third kind will reduce the probability of survival of junior adults by more for the well endowed than for the poorly endowed ones. An epidemic of the second kind has the opposite effect.

The elasticities of the probability of survival of the junior adults with respect to the parameters of this function, are $\quad[\partial \pi(l) / \partial B] /[\pi(l) / B]=B l /\left(B l+B^{\prime}\right), \quad$ and $\left[\partial \pi(l) / \partial B^{\prime}\right] /\left[\pi(l) / B^{\prime}\right]=B^{\prime} /\left(B l+B^{\prime}\right)$. We deduce from these expressions and Lemma 2 that an epidemic of the third kind will reduce the probability of survival of junior adults by a higher proportion for the well endowed than for the poorly endowed ones. An epidemic of the second kind has the opposite effect. Of course, a first kind epidemic as a composite epidemic, will reduce the probability of survivals of well endowed and poorly endowed junior adults by the same proportion. We have a similar result for the effects of an epidemic hitting children on their probability of survival, and their dependence on the endowment of their parents.

We remind that a junior adult is wholly altruistic, and that his utility is proportional to the expected human capital accumulated by his children who will reach the adult age: $U\left(l, e_{+1}\right)=\lambda\left(e_{+1}\right)[\pi(l) r+1]$. We can draw the indifference curves of this utility function in the plan $\left(l, e_{+1}\right)$. An (anticipated) composite epidemic hitting children and such that the two parameters of their survival function are reduced by the same proportion, decreases their probability of survival $\lambda\left(e_{+1}\right)$ by the same percentage for all values of the spending on their health $e_{+1}$. Thus, the indifference curves of the parent are unchanged although each of them will be associated with a lower value of utility. Consequently, the junior adult will not change the allocation of his health spending between himself and his children when he learns that his children will be hit by a composite epidemic. 
Similarly, an epidemic hitting junior adults and such that the two parameters of their survival function are reduced by the same proportion, decreases their probability of survival $\pi(l)$ by the same percentage for all values of the spending on their health $l$. However, the utility of the junior adult will be reduced by a proportion, which will increase with the spending on his own health. Thus, the slopes of the indifference curves will become less steep. Consequently, the junior adult will reduce his spending on his own health (and will increase the spending on the health of his children).

Finally, parents will have to bring up more children if the number of their offspring $n$ increases. So, the death of a junior adult will create more orphans and its consequence will have become worse. This should imply a transfer of health spending from the whole of the children to their parents. However, under this transfer, health spending per child decreases first because total health spending on children has decreased, secondly because there are more children. This directly reduces the probability of survival of each child. The first effect is the stronger, except when parameter $A^{\prime}$ is zero, when these effects exactly balance.

\subsection{Demographic variables}

The population alive in period $t$ includes $N^{2+}$ and $N^{2-}$ junior adults with human capital endowments respectively equal to $h^{+}$and $h^{-}$. It also includes $N^{3+}$ and $N^{3-}$ senior adults. Finally, it includes $N^{1+}, N^{1-}$ children who have parents with respective human capital $h^{+}$, $h^{-}$, and $N^{10+}, N^{10-}$ orphans with respectively high and low bequests. The parents of the two first kinds of children are the senior adults of the period. So, we have:

$$
\text { (14) } N^{1+}=n N^{3+} \text { and } N^{1-}=n N^{3-}
$$

The populations $N^{10+}, N^{10-}, N^{2+}, N^{2-}, N^{3+}$ and $N^{3-}$ are predetermined in period $t$. The number of well-endowed (poorly-endowed) senior adults which will be alive in period $t+1$ is equal to the number of junior adults with the same endowment who are alive in period $t$, time their rate of survival

$$
\text { (15) } N_{+1}^{3+}=\pi\left(l^{+}\right) N^{2+}, N_{+1}^{3-}=\pi\left(l^{-}\right) N^{2-}
$$

If we use equation (14) in period $t+1$ (notice that the total number of children in this period is equal to the number of junior adults in period $t$ times $n$ ), we get the equations

$$
\text { (16) } N_{+1}^{10+}=n N^{2+}-n N_{+1}^{3+} \text { and } N_{+1}^{10-}=n N^{2-}-n N_{+1}^{3-}
$$


The numbers of well-endowed and poorly-endowed junior adults in period $t+1$ are

$$
\text { (17) } \begin{aligned}
N_{+1}^{2+} & =\lambda\left(e^{+}\right)\left(p N^{1+}+q c N^{10+}\right)+\lambda\left(e^{-}\right)\left(p N^{1-}+q c N^{10-}\right) \\
N_{+1}^{2-} & =\lambda\left(e^{+}\right)\left(N^{1+}+c N^{10+}\right)+\lambda\left(e^{-}\right)\left(N^{1-}+c N^{10-}\right)-N_{+1}^{2+}
\end{aligned}
$$

\subsection{Balance of trade and international borrowing}

In period $t$, human capital in the country is equal to $N^{2+} h^{+}+N^{2-} h^{-}$. This expression also gives the quantity of health good domestically produced that is domestic output. The national demand for health good is $N^{2+} l^{+}+N^{2-} l^{-}+\left(N^{1+}+N^{10+}\right) e^{+}+\left(N^{1-}+N^{1 \grave{a}-}\right) e^{-}$. The excess of supply over demand is equal to the surplus of the trade balance $B T$. If we use equations (1), (2), (14), (15) and (16) we can write this surplus as

(18) $B T=\left(N^{2+} s^{+}+N^{2-} s^{-}\right)-\left(N^{3+} s_{-1}^{+}-N^{3-} s_{-1}^{-}\right) R-\left\lfloor N_{-1}^{2+}\left(1-\pi\left(l_{-1}^{+}\right)\right) s_{-1}^{+}+N_{-1}^{2-}\left(1-\pi\left(l_{-1}^{-}\right)\right) s_{-1}^{-}\right\rfloor R$

The first term represents saving by junior adults. The second term represents the disaving (interests included) by senior adults. The last term represents the disaving of the dead, or if one prefers by the orphans.

If we use equation (13) this expression can be rewritten

$$
\text { (19) } B T=\left(N^{2+} s^{+}+N^{2-} s^{-}\right)-\left(N_{-1}^{2+} s_{-1}^{+}+N_{-1}^{2-} s_{-1}^{-}\right) R
$$

The second term of the right-hand side represents assets held by nationals at the beginning of period $t$. The first term represents assets held by nationals at the end of period $t$. They will be inflated by the factor $R$ at the beginning of period $t+1$. Thus, national assets grow at the same rate as the population of junior adults. We will show that in the steady state this rate is lower than $n$, the number of children by junior adults, and we will assume that $n<R$. So, the discounted value of national assets (debt) will tend to 0 when time increases indefinitely.

\section{Dynamics and long run equilibrium}

We will start by examining the equations giving the dynamics of populations. Then, in a second paragraph, we will investigate the properties of this dynamics, when the environment of the economics is kept unchanged.

\subsection{The dynamics of populations}


There are $N^{2+}$ and $N^{2-}$ junior adults alive in period $t \geq 0$. They will have $n$ children each. These children will either become $N_{+2}^{2+}$ and $N_{+2}^{2-}$ junior adults with earnings respectively equal to $h^{+}$and $h^{-}$in period $t+2$, or they will die at the end of period $t+1 . D_{+2}$ represents the supplementary number of junior adults who would exist in period $t$ if no children die before reaching the age of junior adult, that is if the survival rate function $\lambda$ were identical to 1. We will investigate the dynamics of the model for $t \geq 2$. The states of the economy in periods 0 and 1 are assumed to be given. We have the fundamental relationship:

$$
\text { (20) }\left(\begin{array}{l}
N_{+2}^{2+} \\
N_{+2}^{2-} \\
D_{+2}
\end{array}\right)=M n\left(\begin{array}{c}
N^{2+} \\
N^{2-} \\
D
\end{array}\right)=\left(\begin{array}{ccc}
a_{11} & a_{12} & 0 \\
a_{21} & a_{22} & 0 \\
1-a_{11}-a_{21} & 1-a_{12}-a_{22} & 1
\end{array}\right) n\left(\begin{array}{c}
N^{2+} \\
N^{2-} \\
D
\end{array}\right)
$$

with

$$
\begin{gathered}
a_{11}=\lambda\left(e_{+1}^{+}\right)\left\{\pi\left(l^{+}\right) p+\left[1-\pi\left(l^{+}\right)\right] c q\right\} \\
a_{21}=\lambda\left(e_{+1}^{+}\right)\left\{\pi\left(l^{+}\right)(1-p)+\left[1-\pi\left(l^{+}\right)\right] c(1-q)\right\} \\
a_{12}=\lambda\left(e_{+1}^{-}\right)\left\{\pi\left(l^{-}\right) p+\left[1-\pi\left(l^{-}\right)\right] c q\right\} \\
\left.a_{22}=\lambda\left(e_{+1}^{-}\right)\left\{\pi\left(l^{-}\right)(1-p)+\left[1-\pi\left(l^{-}\right)\right] c(1-q)\right\}\right)
\end{gathered}
$$

and with $N^{2+}(0), N^{2-}(0)$ and $D(0)$ given if $t$ is even and $N^{2+}(1), N^{2-}(1)$ and $D(1)$ given if $t$ is odd.

Lemma 1,2 and 3 imply that these parameters satisfy the constraints $0<a_{12}<a_{11}<1$, $0<a_{22}<a_{21}<1, \quad a_{12}+a_{22}<a_{11}+a_{21}<1$ and $a_{11} a_{22}-a_{12} a_{21}=c(p-q) \lambda\left(e_{+1}^{+}\right) \lambda\left(e_{+1}^{-}\right)\left[\pi\left(l^{+}\right)-\pi\left(l^{-}\right)\right\rfloor>0$.

The elements of each column of $M$ are positive and sum to 1 . So they can be interpreted as proportions, or as conditional probabilities for instance for a child of a well-endowed junior adult to be well-endowed or poorly-endowed or dead two periods later.

More precisely, $a_{11}-a_{12}$ is the difference between the probabilities for a child to reach a high level of human capital if his parent is well-endowed versus if his parent is poorly-endowed. $a_{21}-a_{22}$ is the difference between the probabilities for a child to reach a low level of human 
capital if his parents are well-endowed versus if his parents are poorly endowed. The difference between the probabilities for a child to die if his parents are well-endowed versus if his parents are poorly endowed is $-\left(a_{11}-a_{12}\right)-\left(a_{21}-a_{22}\right)$. The fate of children is independent of the social position of their parents when $a_{11}-a_{12}=a_{21}-a_{22}=0$.

Matrix $M$ in period $t$ only depends on health spending set by junior adults, $l^{+}, l^{-}, e_{+1}^{+}$and $e_{+1}^{-}$. These spending are functions of the values taken by a series of exogenous variables in period $t$ : the foreign interest rate $R$, the parameters of the survival functions of children and young adults $A, A^{\prime}, B, B^{\prime}, \alpha$ and $\beta$, the incomes of the junior adults $h^{+}$and $h^{-}$and the number of their children $n$.

Equation (20) gives the dynamics of the numbers of junior adults and of the dead, $\mathrm{N}^{2+}, \mathrm{N}^{2-}$ and $D$ for $t \geq 2$, when the values of these variables are given in periods 0 and 1 . Equation (15) gives the dynamics of the numbers of senior adults $N^{3+}=\pi\left(l^{+}\right) N_{-1}^{2+}, \quad N^{3-}=\pi\left(l^{-}\right) N_{-1}^{2-}$ for $t \geq 1$. Equation (14) gives the dynamics of the number of non orphan children $N^{1+}=n N^{3+}$ and $N^{1-}=n N^{3-}$ for $t \geq 1$. Finally, the numbers of orphans in period $t \geq 1$ are given by equations (16) $N_{+1}^{10+}=n N^{2+}-n N_{+1}^{3+}$ and $N_{+1}^{10-}=n N^{2-}-n N_{+1}^{3-}$.

We define $P=N^{2+}+N^{2-}+D$ as the potential population of junior adults. It would be equal to the effective population if all children reached the age of junior adult. Equation (20) shows that this potential population grows at rate $n: P_{+2}^{2}=n P^{2}$. The number of dead people is equal to the difference between the potential population and the number of junior adults: $D=P-\left(N^{2+}+N^{2-}\right)$. Thus, we just have to investigate the dynamics of the numbers of living junior adults $\mathrm{N}^{2+}$ and $\mathrm{N}^{2-}$, which is given by

(21) $\left(\begin{array}{l}N^{2+}(t+2) \\ N^{2-}(t+2)\end{array}\right)=M^{\prime} n\left(\begin{array}{l}N^{2+}(t) \\ N^{2-}(t)\end{array}\right)=\left(\begin{array}{ll}a_{11} & a_{12} \\ a_{21} & a_{22}\end{array}\right) n\left(\begin{array}{l}N^{2+}(t) \\ N^{2-}(t)\end{array}\right)$

with $N^{2+}(0)$ and $N^{2-}(0)$ given if $t$ is even and $N^{2+}(1)$ and $N^{2-}(1)$ given if $t$ is odd. In the rest of the paper we will assume that $t$ is even.

\subsection{Characterization of the demographic dynamics}

We will assume in this section that all the parameters and exogenous variables stay constant over time for $t \geq 0$. We will also assume that $t$ is even. Then, matrix $M$ will stay constant 
over time, and the dynamics of the model will be limited to the sizes of the various components of population (including the dead). Let us introduce the new variable

$$
\text { (22) } \Delta \equiv\left(a_{11}+a_{22}\right)^{2}-4\left(a_{11} a_{22}-a_{12} a_{21}\right)=\left(a_{11}-a_{22}\right)^{2}+4 a_{12} a_{21}>0
$$

We have the lemma

Lemma 4. a) The eigenvalues of matrix $M^{\prime}, \rho_{1}$ and $\rho_{2}$, are real and such that $1>\rho_{1}>\rho_{2}>0$. Their expressions are

$$
\text { (23) } \rho_{1}=\left(a_{11}+a_{22}+\sqrt{\Delta}\right) / 2 \text { and } \rho_{2}=\left(a_{11}+a_{22}-\sqrt{\Delta}\right) / 2
$$

b) Let us denote by $V_{1}=\left(\begin{array}{l}v_{11} \\ v_{21}\end{array}\right)$ and $V_{2}=\left(\begin{array}{l}v_{12} \\ v_{22}\end{array}\right)$ the right-hand column eigenvectors of $M^{\prime}$ and by $V=\left(\begin{array}{ll}V_{1} & V_{2}\end{array}\right)$ the matrix of these eigenvectors. A determination of these eigenvectors is

$$
\text { (24) } V=\left(\begin{array}{cc}
2 a_{12} & -2 a_{12} \\
a_{22}-a_{11}+\sqrt{\Delta} & -a_{22}+a_{11}+\sqrt{\Delta}
\end{array}\right)
$$

$V_{1}$ can be normed such that its components are positive and sum to $1 . V_{2}$ can be normed such that its first component is negative, its second component is positive and the sum of both components is equal to 1 .

c) Let $W=\left(\begin{array}{ll}w_{11} & w_{12} \\ w_{21} & w_{22}\end{array}\right)$ be the inverse of $V: V W=I$. Then, we have

$$
\text { (25) } W=\frac{1}{4 a_{12} \sqrt{\Delta}}\left(\begin{array}{ll}
-a_{22}+a_{11}+\sqrt{\Delta} & 2 a_{12} \\
-a_{22}+a_{11}-\sqrt{\Delta} & 2 a_{12}
\end{array}\right)
$$

d) The elements of matrix $W$ satisfy the constraints

$$
\text { (26) } w_{11}>w_{12}>0 \text { and } w_{21}<0<w_{22}
$$

The proof is in the appendix. We can now establish the following crucial proposition which neatly characterizes the demographic dynamics and the evolution of human capital (and thus income) distributions over time. 
Proposition 1. Assume, to fix the ideas, that $N^{2+}(0)+N^{2-}(0)=1$. Then:

a) The dynamic paths followed by the sizes of the cohorts of both kinds of junior adults, are linear combinations of two geometric series with rates equal to the growth rate of potential population $n$ times the eigenvalues of matrix $M^{\prime}$

(27) $N^{2+}(t+2)=\left(\rho_{1} n\right)^{t / 2+1} v_{11}\left[w_{11} N^{2+}(0)+w_{12} N^{2-}(0)\right]+\left(\rho_{2} n\right)^{t / 2+1} v_{12}\left[w_{21} N^{2+}(0)+w_{22} N^{2-}(0)\right]$

(28) $N^{2-}(t+2)=\left(\rho_{1} n\right)^{t / 2+1} v_{21}\left[w_{11} N^{2+}(0)+w_{12} N^{2-}(0)\right]+\left(\rho_{2} n\right)^{t / 2+1} v_{22}\left[w_{21} N^{2+}(0)+w_{22} N^{2-}(0)\right]$

In the long run the populations of both kinds of junior adults will grow at a rate equal to the growth rate of the potential population of junior adults times the largest eigenvalue of matrix $M^{\prime}$ (which is smaller than 1). The long run size of each group depends on the initial condition, $\mathrm{N}^{2+}(0)$. However, the long run proportions of the two groups of junior adults are independent of the initial conditions, and are precisely proportional to the two components of the eigenvector associated to the largest eigenvalue of matrix $M^{\prime}$.

b) Let us assume that its share of junior adults holding a high level of human capital in the initial population is decreased. In the long run, the sizes of both groups of junior adults will drop. In the short run, the number of junior adults holding a high level of human capital and the total size of the population of junior adults will unambiguously go down. In contrast, the number of junior adults holding a low level of human capital may increase in the short run.

The proof is in the appendix. Proposition 1 has several important implications, which will be illustrated later on in our application to epidemics next section. First of all, Property a) shows the ability of the model to generate hysteresis. This should not be though seen as a surprising result: this is a natural outcome in demographic models: initial demographic shocks are likely to have long lasting echo effects. Such effects may be dampened after a while, for example if fertility markedly changes some generations after the initial shock, but it seems out of question that persistence is a fundamental property of demographic dynamics. Second, our model features that an initial change in the income distribution of the population may distort this distribution in the short and medium terms but not in the long run. This is a very important property as we will see in the application to epidemics. Actually, one of the debates around AIDS (especially in sub-Saharan Africa) is its impact on income inequality either in the short or long run. Our benchmark model delivers a very clear message in this respect as explained hereafter. 


\section{The demographic and economic effects of epidemics}

We define an epidemic as an increase in the death rate of a generation of people lasting for only one period. Two kinds of epidemics will be considered in this paper. We shall study analytically in detail the impact of epidemics of the first kind, that is those lowering simultaneously and by the same magnitude the two parameters of the survival probabilities ( $A$ and $A^{\prime}$ for children, and $B$ and $B^{\prime}$ for young adults). We then move to the epidemics of the second kind, which we analyze qualitatively so as to unburden the exposition. As one can deduce from Lemma 2 and Lemma 3, the dynamics induced by an epidemic of the third kind are qualitatively very similar to those following an epidemic of the first kind, at least for epidemics hitting young adults. Therefore, we disregard them.

An epidemic hits people irrespectively of their endowment in human capital or of their social background. ${ }^{7}$. We will assume that nothing can be done against the epidemic itself, although an increase in health spending will reduce the number of death the epidemic causes. Finally, we shall only consider one-period long epidemics occurring in period 0 . Longer epidemiological shocks would complicate tremendously the analytical treatment. As we shall see, one-period long shocks are enough to capture the main mechanisms at work in the model and to identify the outcomes of an epidemic of a given kind and age-profile of mortality.

In all cases, we will start from a reference balanced growth path with a total population of junior adults equal to 1 . We first define precisely such a balanced path. We can deduce from the expressions of matrices $V$ and $W$ given in Lemma 4 that $w_{11} v_{11}+w_{12} v_{21}=1$, and $w_{21} v_{11}+w_{22} v_{21}=0$. Assume that the initial population of junior adults, $N^{2+}(0)+N^{2-}(0)$, is equal to 1 , and suppose we norm eigenvector $V_{1}$ in such a way that the sum of its two components is equal to 1 . If the vector of the initial values of the populations of the two kinds of junior adults is equal to the eigenvector of the transition matrix associated to its largest eigenvalue $\left(\begin{array}{l}N^{2+}(0) \\ N^{2-}(0)\end{array}\right)=V_{1}$, the population of junior adults will follow the balanced growth path

\footnotetext{
${ }^{7}$ The assumption that the reduction in the probability of survival is the same for junior adults with a high as with a low level of human capital is debatable. There are indications that people with a relatively high schooling level are more exposed to the risk of being hit by AIDS because they have more sexual partners (Cogneau and Grimm, 2005). There are also indications that these people are more aware of the risks of AIDS than less educated people and understand faster the usefulness of not engaging in risky behaviour, for instance they are more responsive to campaigns of information, and prevention (de Walque, 2004).The United Nations (2004) quotes several studies showing that poor and uneducated people are more likely to engage in risky behaviour and to acquire HIV/AIDS.
} 


$$
\text { (29) }\left(\begin{array}{l}
N^{2+}(t+2) \\
N^{2-}(t+2)
\end{array}\right)=\left(\rho_{1} n\right)^{t / 2+1} V_{1}
$$

Proposition 1 shows that this steady state is relatively asymptotically stable. This will be our reference balanced growth path. We now move to our analysis of epidemics. For a better understanding, recall that total domestic output in our model is given by

$$
\text { (30) } Y(t)=N^{2+}(t) h^{+}+N^{2-}(t) h^{-} \text {. }
$$

\subsection{Epidemics of the first kind}

\subsubsection{An epidemic hitting children}

The epidemic takes place in period 0 and kills a given proportion of children. So, the population of junior adults alive in period 1 will be reduced by the same proportion. However, the ratio between the numbers of well-endowed and poorly endowed junior adults will be unchanged. The second effect will be that the population of junior adults will be reduced by a constant proportion in every odd period by the children, grandchildren, etc. who will not be born because of the death of their forebear. Domestic output will be reduced by the same proportion in odd periods.

Let us investigate the problem at a more formal level. The value of parameters $A$ and $A^{\prime}$ are decreased in a way such that $d A / A=d A^{\prime} / A^{\prime}<0$ in period 0 . Under this assumption, even if the epidemic has been perfectly anticipated when junior adults set their investment decisions, they would have not changed these decisions in front of this information. Equations (20) and (21) show that matrix $M^{\prime}$ is reduced by a factor $(1-\alpha) d A / A$ in period 0 . So, the populations of both kinds of junior adults in every odd period starting in period 1 is reduced by the same proportion. These populations remain unchanged in even periods.

Equations (14), (15) and (16) show that in odd periods the numbers of senior adults and of children of each category, are unchanged. These numbers are reduced by the factor $(1-\alpha) d A / A$ in even periods starting in period 2 . The only demographic change in period 0 is the death of children caused by the epidemic. Thus, the third consequence of the epidemic of period 0 is an echo effect, which permanently changes the demographic structure of the population. The share of junior adults is reduced in every odd period and increased in every even period. Thus, even if domestic output per worker remains the same in these periods, domestic output per capita decreases in odd periods and increases in even periods. 
As we can see, such an epidemic has some important demographic and economic effects either in the short or long run by inducing a permanent demographic composition effect and a change in output per capita (but not per worker). Nonetheless, the epidemic is shown to be neutral at all temporal horizons in terms of the income distribution among junior adults. The next section shows that 'adult' epidemics can in contrast distort such a distribution.

\subsubsection{An epidemic hitting junior adults}

The epidemic takes place in period 0 and kills a proportion of junior adults at the end of the period. The number of children alive in period 1 will be unchanged but the proportion of orphans among them will be higher. The number of senior adults alive in period 1 will be lower as a result of the epidemic.

Let us investigate the problem at a more formal level. Since the epidemic is of the first kind, we are in the situation in which $B$ and $B^{\prime}$ are decreased in a way such that $d B / B=d B^{\prime} / B^{\prime}<0$ in period 0 . Junior adults living in this period perfectly understand the consequences of the epidemic when they make their decisions. According to lemma 2, they will reduce their investment in their own health, and their survival rates at the end of the period will decrease by more than what results from the epidemic. Junior adults will also increase their investment in the health of their children in period 1, which will improve the survival rates of children in period 1. Thus, matrix $M^{\prime}$ has been changed in period 0 , and consequently the populations of junior adults in period 2. The relative variations in the populations of juniors adults holding a high level and a low level of human capital, in this period is

$$
\begin{aligned}
& \text { (31) } \frac{d N^{2+}(2)}{N^{2+}(2)}=\frac{v_{11} d a_{11}+\left(1-v_{11}\right) d a_{12}}{\rho_{1} v_{11}} \\
& \text { (32) } \frac{d N^{2-}(2)}{N^{2-}(2)}=\frac{v_{11} d a_{21}+\left(1-v_{11}\right) d a_{22}}{\rho_{1}\left(1-v_{11}\right)}
\end{aligned}
$$

The relative changes in the total population of junior adults and in the domestic output per worker are

$$
\text { (33) } \frac{d N^{2+}(2)+d N^{2-}(2)}{N^{2+}(2)+N^{2-}(2)}=\frac{v_{11}\left(d a_{11}+d a_{21}\right)+\left(1-v_{11}\right)\left(d a_{12}+d a_{22}\right)}{\rho_{1}}
$$




$$
\begin{aligned}
& \frac{d Y(2)}{N^{2+}(2) h^{+}+N^{2-}(2) h^{-}}-\frac{d N^{2+}(2)+d N^{2-}(2)}{N^{2+}(2)+N^{2-}(2)}= \\
& {\left[N^{2+}(2) N^{2-}(2)\left(h^{+}-h^{-}\right)\right.} \\
& {\left[N^{2+}(2) h^{+}+N^{2-}(2) h^{-} \llbracket N^{2+}(2)+N^{2-}(2)\right]\left[\frac{d N^{2+}(2)}{N^{2+}(2)}-\frac{d N^{2-}(2)}{N^{2-}(2)}\right]}
\end{aligned}
$$

The following lemma is an extension of lemmas 2 and 3.

Lemma 5. Let us consider a junior adult with endowment $h$, and a decrease in the coefficients of his survival function by $d B / B=d B^{\prime} / B^{\prime}<0$. His probability of survival and the probability of survival of each of his children will change by

(35) $\frac{d \pi(l)}{\pi(l)}=(1-\beta) \frac{\left[n B A^{\prime} /(A R)+B^{\prime}+B h\right] /\left(B l+B^{\prime}\right)}{\beta\left[n B A^{\prime} /(A R)+B^{\prime}+B h\right] /\left(B l+B^{\prime}\right)+2-\alpha-\beta} \frac{d B}{B}<(1-\beta) \frac{d B}{B}<0$

$$
\text { (36) } \begin{aligned}
& \frac{d \lambda\left(e_{+1}\right)}{\lambda\left(e_{+1}\right)}=-(1-\alpha) \frac{B l+B^{\prime}}{B(h-l)+n B A^{\prime} /(A R)} \frac{\frac{(1-\alpha)(1-\beta)}{r\left(B l+B^{\prime}\right)^{1-\beta}}}{\left(\beta \frac{n B A^{\prime} /(A R)+B^{\prime}+B h}{B l+B^{\prime}}+2-\alpha-\beta\right)} \frac{d B}{B}= \\
& -\frac{\left[(1-\alpha)\left(B l+B^{\prime}\right)\right]^{2}}{\left[B(h-l)+n B A^{\prime} /(A R)\right]\left[n B A^{\prime} /(A R)+B^{\prime}+B h\right]} \frac{1}{(1-\beta) r \pi(l)} \frac{d \pi(l)}{\pi(l)}>0
\end{aligned}
$$

Proof. See the appendix.

An epidemic decreases the probability of survival of junior adults, first because it increases the death rate of this population, secondly because it reduces the spending of this population on its own health. This epidemic increases the probability of survival of children (conditionally on the facts that they are orphans or that their parents are alive) because parents spend more on the health of their children. The following lemma will be used in the proof of Proposition 2.

Lemma 6. Consider a junior adult with endowment $h$ who invests $l$ in his own health. When parameters $c$ and $q$ change, the expression

$$
\begin{aligned}
& E=\frac{(1-\alpha)\left(B l+B^{\prime}\right)}{B h+B^{\prime}+n B A^{\prime} /(A R)}\left[1-\frac{1-\alpha}{1-\beta} \frac{B l+B^{\prime}}{B(h-l)+n B A^{\prime} /(A R)}\right] \\
& =\frac{\left[(1-\alpha)\left(B l+B^{\prime}\right)\right]^{2}}{\left[B(h-l)+n B A^{\prime} /(A R)\right]\left[n B A^{\prime} /(A R)+B^{\prime}+B h\right]} \frac{1}{(1-\beta) r \pi(l)}
\end{aligned}
$$


has a positive lower bound $\underline{E}$ and an upper bound $\bar{E}$ smaller than 1.

Proof. See the appendix.

The following proposition will give the changes, taking place in period 2 , in the total population of junior adults, and in the population of workers holding, respectively, a high level and a low level of human capital, induced by an epidemic taking place in period 0 .

Proposition 2. If the reduction in the probability of survival of orphans, $1-c$, and if the probability for an orphan to reach a high level of human capital, $q$, are low enough, we have the following results.

a) In period 2 the total population of junior adults increases.

b) The population of junior adults holding a high level of human capital decreases, and the population of junior adults with a low level of human capital increases. Thus, the proportion of junior adults with a low endowment of human capital in the total population increases. Consequently, domestic output per worker decreases.

c) The numbers of each kind of children and senior adults are unchanged.

The proof is in the appendix. When an epidemic takes place, well-endowed junior adults will spend more on the health of their children. This will contribute to increasing the proportion of these children who will survive in period 2. However, more of these children will grow as orphans whose the probability of survival is reduced by a factor $1-c$. If $c$ is near enough to 1 , the first effect will dominate and the number of junior adults alive in period 2 will be higher.

In period 2, the number of junior adults who were orphans will increase and the number of those who were brought up by their parents will decrease. If the probability for an orphan to reach a high level of human capital, $q$, is low enough, the number of junior adults with a high level of human capital, alive in period 2, will become lower. The two assumptions of Proposition 2 mean that orphans are more disadvantaged in their probability of reaching a high level of human capital than in their probability of dying before adult age.

Proposition 2 is a crucial characterisation of the medium term distributional effects of 'adult' epidemics. In contrast to the epidemic only killing children, considered before, the 
distributional consequences are significant in the medium run. More young adults will get less educated two periods after the epidemic and output per worker goes down: the economy is clearly impoverished (with respect to the reference balanced growth path) at this time horizon $^{8}$. Thus, the demographic and economic effects are clearly much more potentially dangerous when the epidemic hits junior adults than when it only affects children.

The analysis of periods posterior to period 2 is cumbersome. We know that, in the long run, the shares of junior adults holding respectively a high level and a low level of human capital that is the income distribution will go back to their balanced growth values. So, in contrast to some contributions in the AIDS-related literature (like Bell et al., 2003), the model predicts a kind of corrective dynamics which will bring some key variables to the corresponding balanced growth corresponding values. But we cannot even conclude on the long run change in the total population of junior adults without further assumptions. However, we can note that just like 'child' epidemics and for the same reasons, we have some permanent effects, notably on the demographic composition of the economy.

\subsection{Epidemics of the second kind}

To unburden the presentation, we shall discuss the implications of the epidemics of the second kind in qualitative terms. Of course, the algebra involved is pretty much similar to the previous analysis.

\subsubsection{Epidemics hitting children}

The dynamics induced will depend closely on whether the parents of the children will anticipate or not in $t=-1$ the shock taking place in $t=0$. In the case where the shock is unanticipated, we get a similar picture as the one depicted in Section 4.1.1 devoted to epidemics of the first kind hitting children. But the shock might be perfectly anticipated: it could be so in the case of a chronic disease (like malaria) or because the economy has been experiencing an epidemic hitting adults before $\mathrm{t}=0$, which is likely to be transmitted to children (like AIDS). Let us isolate here the impact of the epidemic hitting children at $\mathrm{t}=0$, anticipated by their parents one period before. In such a case, as documented in Lemma 2 and Lemma 3, the parents will spend less on their own health and more on the health of their children. This implies less senior adults in $\mathrm{t}=0$ and more orphans too. In $\mathrm{t}=1$, the number of

\footnotetext{
${ }^{8}$ However, the share of the active population in the total population increases and we do not know if output per capita increases or decreases.
} 
senior adults is unchanged, and so is the number of children in the same period (since the number of young adults in $\mathrm{t}=0$ is not affected by the epidemic). However, the number of young adults generally diminishes in $t=1$. In effect, more orphans in $t=0$ means less young adults in $\mathrm{t}=1$, and such an induced drop should be added to the direct effect of the epidemic killing a proportion of children in $\mathrm{t}=0$. On the other side, if the parents anticipate the epidemic in $\mathrm{t}=0$ and spend more on the health of their children, then the survival probability of children is higher, which tends to increase the number of junior adults in $t=1$. The latter effect is generally dominated by the two former negative effects, including the direct one. Henceforth, the number of junior adults should drop in period $t=1$, leading to a further drop in the number of children and senior adults in $t=2$. However, the number of junior adults in unchanged in period $\mathrm{t}=2$, since the number of children at $\mathrm{t}=1$ and their survival probability is unaffected by the initial shock and its further consequences.

Therefore except the fact that the number of senior adults should also drop in $t=0$ in contrast to the epidemic of the first kind hitting children for which such a figure starts to fall from $t=2$, we have qualitatively the same kind of demographic dynamics as in Section 4.1.1: The epidemics of the first and second kind hitting children at $\mathrm{t}=0$ induce a permanent demographic restructuring with the proportion of junior adults rising in even periods and decreasing in odd periods.

Nonetheless, in sharp contrast to the epidemic of the first kind, the current epidemic configuration has short and medium term distributional effects. Even fully anticipated, an epidemic of the first kind hitting children will not affect the income distribution because the investment decisions of the junior adults are unaltered. However, an epidemic of the second kind does alter such investment decisions if it is anticipated one period behind: junior adults at $\mathrm{t}=-1$ will invest more on the health of their children and less on themselves. The situation is quite similar to the one algebraically investigated in Section 4.1.2 above which impact two periods after the shock is summarized by Proposition 2: under the same conditions, that it is if orphans are more penalized in the access to a high level of human capital than in terms of survival rates, the proportion of unskilled junior adults or workers will drop in $t=1$, leading to the fall of output per worker in this period.

Thus while the two epidemics share almost the same qualitative demographic impact, the short and medium term economic and distributional are quite different in the case where the epidemics are anticipated by the parents. In particular, an anticipated epidemic of the second 
kind hitting children has a negative distributional effect in $\mathrm{t}=1$ while the epidemic of the first kind, even fully anticipated, has none.

\subsubsection{Epidemics hitting young adults}

In contrast to epidemics of the first kind hitting young adults at $\mathrm{t}=0$, the latter will not react by cutting their health expenditures and increasing those of their children: they will do just the contrary. Nonetheless, this will not reverse all the results stated in Proposition 2. In effect, even if the young adults increase their health expenditures, the induced gain in life expectancy is generally not sufficient to compensate the decrease in the number of young adults directly caused by the epidemic in $\mathrm{t}=0$. Henceforth, while we will have more young adults surviving in $\mathrm{t}=1$ and therefore less orphans compared to the case studied in Section 4.1.2, we will still have less senior adults and more orphans in $\mathrm{t}=1$ compared to the benchmark balanced growth path. The situation is definitely worse in $\mathrm{t}=2$ : in contrast to "adults" epidemics of the first kind, since the young adults in $\mathrm{t}=0$ will invest less in the health of their children, we will have unambiguously less junior adults in $\mathrm{t}=2$. The proportion of unskilled junior adults will also increase (compared to the balanced growth path) exactly as in epidemic shocks studied in the two previous sections, which lowers again output per worker in $\mathrm{t}=2$.

It seems therefore crystal clear that the epidemics of the second kind generally lead to a more negative evolution either from the demographic or economic point of view. In particular, a striking difference between the two epidemics is that while the epidemic of the first kind increases the number of junior adults in $\mathrm{t}=2$, the epidemic of the second kind induces just the contrary. It is now time to switch to a more factual evidence to substantiate the discussion.

\subsection{Discussion}

In order to give a focused discussion, we shall concentrate on the short and medium-term dynamics, say for example the outcomes at $t=1,2$. The long-run equilibrium has been already studied in Section 3.2, and its properties summarized in Proposition 1. We shall also start with some demographic projections in the AIDS case to discriminate between the theoretical cases studied above. $^{9}$

\footnotetext{
${ }^{9}$ By doing so, we have not in mind any kind of calibration or purposive empirical work. Of course, the fact that for analytical tractability we only consider one-period shocks does not allow for such a task.
} 


\subsubsection{Demographic projections in the AIDS case}

Fortunately, the disagreement on the economic impact of AIDS does not extend to the demographers, at least for the medium term horizon. HIV/AIDS primarily affects the most productive age group of men and women between 15 and 49 years-the main breadwinners and heads of households raising families and supporting the elderly-and their children. All studies conclude that the total population of countries severely hit by AIDS will be much lower in 20 or 25 years than if the epidemic had not taken place. Figure 1 (United Nations, $2004^{10}$ ) presents the projected population size from 1995 to 2025, taking into account the demographic impact of AIDS as well as the hypothetical projected population without AIDS, for the 38 most affected African countries. In 1995, their population stood at 553 million, 6 million less than it would have been without AIDS. By 2025, the population of these 38 African countries will reach 983 million, that is, 156 million (or 14 per cent) fewer than without AIDS. This number can be decomposed between 98 million additional deaths between 1995 and 2025, and 58 million children who will not be born because of the early deaths of women of reproductive age. In the most severe case, Botswana, where currently more than one in three adults is HIV positive, population is expected to decline within the next few years

Death affects more the adult population of working age than younger or older populations. However, the same study by the United Nations writes "Approximately one fourth to one third of children born to HIV-positive women are likely to acquire infection from their mothers. Paediatric HIV infection is expected to have a substantial impact on mortality during infancy and childhood, particularly among older children (above age one)... Children who acquire the HIV virus from their mothers during childbirth or breast feeding usually do not survive long

\footnotetext{
${ }^{10}$ We can read in this report: "In World Population Prospects: The 2002 Revision (United Nations, Sales $\mathrm{N}^{\circ}$ E.03XIII.6), the United Nations Department of Economics and Social Affairs Population Division incorporated the impact of AIDS into the estimates and projections of the population of 53 countries... They are compared with hypothetical estimates and projections that make no allowance for the existence of AIDS. The latter are derived from the application of the Population Division standard projection program on the basis of assumptions regarding the future course of mortality that are similar to and consistent with those made with respect to countries that are still largely free from the HIV/AIDS epidemic. The process to derive estimates and projections that explicitly incorporate the effect of HIV/AIDS is more complex and made in several steps... In many of the investigated countries, the prevalence of HIV was still rising at the time of the most recent observation. In most such cases, the projections assume that HIV prevalence will peak sometimes during the period 2002-2020. In about half of the countries, the peak prevalence is estimated to have occurred already, between 1993 and 2001. However, in some of those cases the evidence remains weak that prevalence had indeed passed its peak... Thereafter, prevalence levels are assumed to decline in a manner consistent with modifications of behaviour that reduce the rates of recruitment into high-risk groups and the chance of infection among those engaging in highrisk behaviour."
} 
enough to enrol in school.... Children die young from HIV owing to mother-to-child transmission and to the weakened ability of infected mothers to care for their infants and young children". Cohen (1998) notes on Southern Africa: "Infant mortality rates are already rising sharply in countries with mature epidemics. Children born to mothers who are HIV positive have a $30-60 \%$ chance of becoming positive themselves... It is estimated that twothirds of AIDS-deaths will occur in children aged 1-4 years... Child mortality rates are already higher today than they would have been without AIDS in some high prevalence countries. Thus child mortality rates are estimated as being 75\% higher in Botswana in 1996. By the year 2010 child mortality rates are expected to be twice as high in Botswana, 4 times greater in Zimbabwe and about twice as high in Zambia and Malawi” (Figure 2).

Finally, Figure 3 (United Nations, 2004) displays the age pyramid of Botswana, the country with the highest adult HIV prevalence, in 2000 and as projected for 2025, with and without AIDS. In 2000, the impact of AIDS on the age structure of Botswana's population is still mild. But by 2025, more than half of the potential population aged 35-59 would have been lost to AIDS. In comparison, one third of the population aged less than 15 years old is expected to be lost to AIDS. Cohen (2002) notes that for Malawi one of the most important consequences of AIDS is a change in the age pyramid of the population, with a narrowing of the distribution in the working age population, and a consequent problem with respect to age dependency, with larger numbers of youthful and elderly dependents.

With these "stylized" demographic facts in mind, we shall examine now the adequacy and predictions of our theory. To this end, we will consider an epidemic hitting simultaneously children and young adults, which will incidentally allow us to provide a synthesis of the properties established in Sections 4.1 and 4.2. To fit even better the demographic exposition just above, we shall consider epidemics hitting more young adults than children, and examine the implications for $\mathrm{t}=1,2$.

\subsubsection{Testing the theory}

We shall test the theory on four points, two demographic aspects (population size, age pyramid), and two economic (output and productivity indicators, income distribution).

a) Population size: Putting together the implications of epidemics of the first kind hitting children and adults at $\mathrm{t}=0$, one gets a quite counter-factual picture. In the short run, say 
$\mathrm{t}=1$, the epidemic hitting children mechanically decreases the number of junior adults while keeping unaltered the numbers of senior adults and children. Therefore, overall the total effect of this epidemic is a decrease in the size of the population at $t=1$. On the other hand, the epidemic hitting junior adults only modifies the number of senior adults in $\mathrm{t}=1$. So putting together both shocks, we have a clearly declining population size in $\mathrm{t}=1$, which is correct. However, in $\mathrm{t}=2$, we get the counter-factual prediction: while the epidemic hitting children decreases the size of the population (both the number of children and senior adults decline whereas the number of junior adults is unaltered), the epidemic hitting adults does the contrary since the number of junior adults gets increased in $t=2$ under this epidemic (See proposition 2). Henceforth, if we assume consistently with the AIDS evidence that the mortality impact of the epidemic is stronger on junior adults than on children, we get a counter-factual overall effect, that is an increase in the population size in the medium-run $(t=2)$, driven by the increase in the number of junior adults in the period, which is itself due to the increase in the investment in the health of children following the shock at the end of the period $\mathrm{t}=0$.

In contrast, the epidemic of the second kind entails the opposite optimal investment response under adult epidemics: more health expenditures for junior adults at the expense of health expenditures on children. As a consequence, the key mechanism yielding the counter-factual prediction above is just reversed, and we get a much more satisfactory picture if we want to replicate AIDS medium-term demographic impact. This property is confirmed in the next point.

b) Age pyramid: In the short run $(t=1)$, the epidemics of the first and second kind both imply a reduction in the proportion of young adults, which is also a key economic implications since these adults are also the workers of the economy. ${ }^{11}$ The crucial difference between the epidemics arises in $\mathrm{t}=2$ for the same reason as before. While the epidemic of the first kind (hitting adults) entails a mechanism inducing an increase in the number of young adults in $\mathrm{t}=2$, and therefore a counter-factual age pyramid, the

\footnotetext{
${ }^{11}$ Note that in our case, this property is a non-trivial consequence of a shock which takes place in the initial period. Naturally, a lasting epidemic like AIDS has additionally a direct effect on the demographic composition at $\mathrm{t}=2$.
} 
epidemic of the second kind does not and fits much better the medium-term age pyramid in Figure 3.

c) Output and productivity: In contrast to the demographic indicators studied above, the epidemics of first and second kind both predict the worsening of economic performance in the short and medium run. This could be easily captured by declining output per capita or output per worker or possibly both in $t=1$ and $t=2$. A nice feature of the theory is that even in the case where the working population is rising, which happens to be the case of adult epidemics of the second kind in period $t=2$, this demographic "advantage" is offset by the increasing proportion of unskilled, which leads output per worker to fall down.

d) Income distribution: The same type of conclusions can be reached for distributional effects. While only the epidemic of the second kind has a (probably slight) distributional effect in the short run, that is $\mathrm{t}=1$, both have such a consequence in the medium term, when $t=2$. Adult epidemics, either of the first or second kind, do trigger an increase in the proportion of unskilled junior adults in $t=2$, which features an unambiguous impoverishment of the economies in the medium run. It should be clearly noted at this point that this property of the model derives from the singularity of a rising category under epidemics, orphans. Under our working assumptions (see in particular Proposition 2), that it is if orphans are more penalized in the access to a high level of human capital than in terms of survival rates, which seems quite reasonable, they will necessarily lead to the medium-term increase in the proportion of unskilled. The recent demographic evidence on the quickly rising number orphans is therefore of crucial importance: if not conveniently treated (for example by internationally funded social aid programs for orphans), this problem is likely to induce a sharp worsening of poverty in the medium run.

\section{Concluding: does fertility matter?}

In our model, we have taken fertility as exogenous. This is done in order to get an analytical representation of demographic and economic dynamics at any period. On a theoretical ground, a persistent decline in life expectancy can increase or decrease fertility depending on parental 
preferences and on the way mortality is specified (see Hazan and Zoaby, 2006, for a purely theoretical argument). Indeed, a sharp increase in child mortality (which happens under AIDS for example) may give rise to an insurance effect leading to a rise in fertility. On the other hand, a Barro-Becker model (1989) where mothers can freely supply labor would feature that under a massive drop in labour supply, female participation in the labor market will shift upward, implying a decrease in fertility. Empirically, the debate is even less clear (see Young, 2005, versus Kalemli-Ozcan, 2006, in the AIDS case). In this paper, we have adopted the viewpoint of most demographers on the fertility behaviour response to AIDS: By the time most HIV infected women die, they would already have given birth to several children. Hence, fertility response to epidemics like AIDS could be actually quite limited. Indeed, many surveys report a lack of awareness of AIDS among the infected people themselves in subSaharan Africa who seldom drastically modify their sexual behavior (see Kalemli-Ozcan, 2006).

Of course, this should not be an excuse to not take the further step of endogenizing fertility in our general theory of dynamics and income distribution under epidemics. Of course, this would be done at the expense of some of the directions taken in the current paper (for example, the tractability of medium term dynamics). But the extension seems highly promising. We shall illustrate its relevance by a simple exercise on the current model.

Suppose that a junior adult living in period 0 has, at the end of this period, a number of children reduced by the amount $d n<0$. In the following periods fertility will be restored to its initial level. We will assume that value of parameter $A^{\prime}$ is zero to make the computations almost immediate. Under this assumption, Lemma 2 and 3 establish that this junior adult will keep his spending on his own health and on the whole of his children unchanged. So, a junior adult with an endowment of human capital $h^{+}$will increase his investment in the health of each of his children by $d e_{+1}^{+}=-e_{+1}^{+} d n / n>0$. The probability of survival of this child will increase by $d \lambda\left(e_{+1}^{+}\right)=(1-\alpha) \lambda\left(e_{+1}^{+}\right) d e_{+1}^{+} / e_{+1}^{+}=-(1-\alpha) \lambda\left(e_{+1}^{+}\right) d n / n>0$.

The number of junior adults alive in period 2 will change in reaction to two opposite effects. It will tend to decrease because of the lower number of children born at the end of period 0 , but it will tend to increase because parents will spend more on the health of each of their children. We can compute the total effect by differentiating equation (21) 


$$
\text { (38) }\left(\begin{array}{l}
d N^{2+}(2) \\
d N^{2-}(2)
\end{array}\right)=\left[M^{\prime} d n+n\left(\begin{array}{ll}
d a_{11} & d a_{12} \\
d a_{21} & d a_{22}
\end{array}\right)\right]\left(\begin{array}{l}
N^{2+}(0) \\
N^{2-}(0)
\end{array}\right)
$$

If we use equation (20) we get

$$
\text { (39) }\left(\begin{array}{ll}
d a_{11} & d a_{12} \\
d a_{21} & d a_{22}
\end{array}\right)=-(1-\alpha) M^{\prime} d n / n
$$

Thus

$$
\text { (40) }\left(\begin{array}{l}
d N^{2+}(2) \\
d N^{2-}(2)
\end{array}\right)=\alpha M^{\prime}\left(\begin{array}{l}
N^{2+}(0) \\
N^{2-}(0)
\end{array}\right) d n=\alpha\left(\begin{array}{l}
N^{2+}(2) \\
N^{2-}(2)
\end{array}\right) d n / n<0
$$

So, the consequence of a decrease in fertility in period 0 will be to reduce the population of both kinds of senior adults in period 2 and every following even period by the proportion $\alpha d n / n$. The ratio between the numbers of well-endowed and poorly endowed junior adults will be unchanged, but the population of junior adults will be reduced by a constant proportion in every even period. The numbers of senior adults and of children of each category will be reduced by the factor $\alpha d n / n$ in odd periods starting in period 3. The only demographic change in period 1 will be the reduction in the number of children resulting from the decrease in fertility. So, the share of junior adults in the total population is reduced in every even period and increased in every odd period. Thus, even if the domestic output per worker remains the same in these periods, domestic output per capita decreases in even periods and increases in odd periods.

In our exercise, the negative fertility shock has a clear negative impact on the size of the population at least in even periods, but no effect at all on income distribution and investment choices. The latter property is not the expected outcome in a Barro-Becker framework where the quantity/quality of children trade-off is key. This should be the next step of our research in the topic.

\section{References}


Barro Robert J. and Gary S. Becker (1989) - "Fertility Choice in a Model of Economic Growth", Econometrica 57(2): 481-501.

Barro Robert J and Xavier Sala-i-Martin (1995) - Economic Growth, Mc Graw-Hill, New York.

Bell Clive, Devarajan Shantayanan and Hans Gersbach (2003) - "The Long-run Economic Costs of AIDS: Theory and Application to South Africa", wps 3152, June.

Blanchard, Olivier (1985) - "Debts, Deficits, and Finite Horizon", Journal of Political Economy 93(2): 223-247.

Bloom, David E. and Ajay S. Mahal (1997) "Does the AIDS Epidemic Threaten Economic Growth?" Journal of Econometrics 77 (1): 105-24.

Boucekkine, Raouf, Bity Diene and Théophile Azomahou (2007), 'The Growth Economics of Epidemics I: Theoretical considerations', Mathematical Population Studies, forthcoming in 2007.

Boucekkine, Raouf, David de la Croix and Omar Licandro (2002), 'Vintage Human Capital, Demographic Trends, and Endogenous Growth', Journal of Economic Theory 104: 340-375.

Bowles Samuel and Herbert Gentis (2002) - "The Inheritance of Inequality", The Journal of Economic Perspective 16(3): 3-30.

Brainerd Elizabeth and Mark V. Siegler (2003) - "The Economic Effects of the 1918 Influenza Epidemic", CEPR Discussion Paper No. 379, February.

Case Anne, Darren Lubotsky and Christina Paxson (2001) - "Economic Status and Health in Childhood: The Origin of the Gradient”, NBER Working Paper 8344. 
Case Anne, Christina Paxson and Joseph Ableidinger (2004) - “Orphans in Africa: Parental Death, Poverty, and school Enrollment”, Demography 41(3): 483-508.

Chakraborty Shanka and Mausumi Das (2005) - "Mortality, Human Capital and Persistent Inequality", Journal of Economic Growth 10(2): 159-192.

Johnson Leigh F. and Dorrington Robb E. (2002) - “The Demographic and epidemiological impact of HIV/AIDS Treatment and Prevention Programmes: An Evaluation Based on the ASSA2000 Model", presented at the 2002 Demographic Association of Southern Africa Conference.

Cogneau Denis and Michael Grimm (2005) - "The Impact of AIDS on the Distribution of Income in Côte d'Ivoire", mimeographed DIAL, Paris, July.

Cohen Desmond (1998) - "Socio-Economic Causes and Consequences of the HIV Epidemic in Southern Africa: A Case Study of Namibia”, Issues Paper $N^{\circ} 31$, HIV and Development Program, UNDP,

Cohen Desmond (2002) - "Human Capital and the HIV Epidemic in sub-Saharan Africa", Working Paper 2, Geneva: ILO Programme on HIV/AIDS and the World of Work.

Corrigan Paul, Gerhard Glomm and Fabio Mendez (2005) - "AIDS Crisis and Growth", Journal of Development Economics 77: 107-124.

Cutler David, Angus Deaton and Ariana Leras-Muney (2005) - "The Determinants of Mortality", mimeographed, Harvard University.

Deaton Angus (2003) - "Health, Inequality, and Economic Development", Journal of Economic Literature, XLI (March): 113-158.

De Walque Damien (2004) - "How Does the Impact of an HIV/AIDS Information Campaign Vary with Educational Attaintment? Evidence from Rural Uganda, Policy Research Working Paper 3289, Washington DC, World Bank. 
Ferreira Pedro C. and Samuel D. Pessoa (2003) - "The Long-Run Economic Impact of AIDS", available at SSRN: http://ssrn.com/abstract=411782.

Galor Oded and Omer Moav (2005) - "Natural Selection and the Evolution of Life Expectancy", mimeographed, November 17.

Galor Oded and Omer Moav (2002) - " Natural Selection and the Origin of Economic Growth”, Quarterly Journal of Economics 117: 1133-1192.

Gertler Paul, David Levine and Sebastian Martinez (2003) - "The Presence and Presents of Parents: Do Parents Matter for More than their Money?" paper presented at the scientific meeting on empirical evidence for the demographic and socio-economic impact of AIDS, hosted by HEARD, Durban, South Africa, 26-28 March.

Grawe Nathan D. and Casey B. Mulligan (2002) - "Economic Interpretation of Intergenerational Correlations", The Journal of Economic Perspective 16(3): 45-58.

Hazan Moshe and Hosny Zoabi (2006) - "Does Longevity Cause Growth? A Theoretical Critique", Journal of Economic Growth 11(4): 363-376.

Hirshleifer Jack (1987) - Economic Behaviour in Adversity, University of Chicago Press, Chicago.

Johnson Leigh F. and Dorrington Robb E. (2002) - "The Demographic and epidemiological impact of HIV/AIDS Treatment and Prevention Programmes: An Evaluation Based on the ASSA2000 Model", presented at the 2002 Demographic Association of Southern Africa Conference.

Kalemli-Ozcan Sebnem (2006) - "AIDS, Reversal of the Demographic Transition and Economic Development: Evidence from Africa”, NBER working paper No. 12181.

Lorentzen Peter, John McMillan and Romain Wacziark (2006) - "Death and Development", Mimeographed, Graduate Scholl of Business, Stanford University. 
Lucas Robert (1988) - "On the Mechanics of Economic Development", Journal of Monetary Economics 22(1): 3-42.

Marseille Elliot, Paul B Hofmann and James G Kahn (2002) - «HIV prevention before HAART in sub-Saharan Africa », Lancet 359: 1851-56.

McDonald Scott and Jennifer Roberts (2006) - "AIDS and Economic Growth: A Human Capital Approach", Journal of Development Economics 80(1): 228-250.

Ricci Francesco and Marios Zachariadis (2006) - "Determinants of Public Health Outcomes: A Macroeconomic Perspective", mimeographed, June.

The Economist (2006) - "Recipe for averting disaster", October $28^{\text {th }}, 95-96$.

The Economist (2003) - “AIDS. A mixed prognosis”, November $29^{\text {th }}, 87-89$.

United Nations (2004) - The Impact of AIDS, Department of Economic and Social Affairs, Population Division, New York.

Young Alwyn (2005) - "The Gift of the Dying: The Tragedy of AIDS and the Welfare of Future African Generations", The Quarterly Journal of Economics 120(2): 423-466. 


\section{APPENDIX}

\section{Proof of Lemma 4}

a) The eigenvalues of matrix $M^{\prime}$ are the roots of the characteristic equation $S(\Lambda) \equiv \rho^{2}-\left(a_{11}+a_{22}\right) \rho+\left(a_{11} a_{22}-a_{12} a_{21}\right)=0$

The discriminant of this equation is $\Delta>0$. So, the two eigenvalues of $M^{\prime}$ are distinct and real. Their product is given by $S(0) \equiv a_{11} a_{22}-a_{12} a_{21}>0$. Moreover we have $S(1) \equiv 1-\left(a_{11}+a_{22}\right)+\left(a_{11} a_{22}-a_{12} a_{21}\right)=\left(1-a_{11}\right)\left(1-a_{22}\right)-a_{12} a_{21}$ As we have $1-a_{11}>a_{21}$ and $1-a_{22}>a_{12}$, we can conclude that $S(1)>0$. Thus, the two eigenvalues of matrix $M^{\prime}$ are strictly included between 0 and 1 .

b) We have

$$
\begin{aligned}
& \left(a_{11}+a_{22}+\sqrt{\Delta}\right) v_{11} / 2=\rho_{1} v_{11}=a_{11} v_{11}+a_{12} v_{21}, \text { so } \\
& \left(a_{22}-a_{11}+\sqrt{\Delta}\right) v_{11}=2 a_{12} v_{21}
\end{aligned}
$$

We also have

$$
\left(a_{22}-a_{11}-\sqrt{\Delta}\right) v_{12}=2 a_{12} v_{22}
$$

So, a determination of the eigenvectors is given by equation (24). The two components of $V_{1}$ are positive and we can norm this eigenvector by setting $v_{11}+v_{21}=1$. Moreover the sum of the two components of $V_{2}$ is positive and we can norm this eigenvector by setting $v_{12}+v_{22}=1$

c) We deduce from $V W=I$

$$
\begin{aligned}
& 2 a_{12}\left(w_{11}-w_{21}\right)=1 \\
& 2 a_{12}\left(w_{12}-w_{22}\right)=0 \\
& \left(a_{22}-a_{11}\right)\left(w_{11}-w_{21}\right)+\sqrt{\Delta}\left(w_{11}+w_{21}\right)=0 \\
& \left(a_{22}-a_{11}\right)\left(w_{12}-w_{22}\right)+\sqrt{\Delta}\left(w_{12}+w_{22}\right)=1 \\
& \text { so } w_{11}=\frac{1}{4 \sqrt{\Delta}} \frac{\sqrt{\Delta}+a_{11}-a_{22}}{a_{12}}>0 \text { and } w_{12}=\frac{1}{2 \sqrt{\Delta}}>0 \\
& \text { and } w_{21}=\frac{1}{4 \sqrt{\Delta}} \frac{-\sqrt{\Delta}+a_{11}-a_{22}}{a_{12}}<0 \text { and } w_{22}=\frac{1}{2 \sqrt{\Delta}}>0
\end{aligned}
$$


d) The inequalities are easy to check. For example, $w_{11}>w_{12}$ is equivalent to $\sqrt{\Delta}>2 a_{12}+\left(a_{22}-a_{11}\right)$. A sufficient condition for this inequality is

$\Delta \equiv\left(a_{11}-a_{22}\right)^{2}+4 a_{12} a_{21}>\left(a_{22}-a_{11}\right)^{2}+4 a_{12}\left(a_{12}+a_{22}-a_{11}\right)$, or

$a_{11}+a_{21}>a_{12}+a_{22}$, which is true.

\section{Proof of Proposition 1}

a) Let $\mathrm{P}$ be the diagonal matrix with elements $\rho_{1}$ and $\rho_{2}$. Then (21) can be rewritten

$$
\left(\begin{array}{l}
N^{2+}(t+2) \\
N^{2-}(t+2)
\end{array}\right)=M^{\prime} n\left(\begin{array}{c}
N^{2+}(t) \\
N^{2-}(t)
\end{array}\right)=V \mathrm{PWn}\left(\begin{array}{c}
N^{2+}(t) \\
N^{2-}(t)
\end{array}\right)=V(n \mathrm{P})^{t / 2+1} W\left(\begin{array}{c}
N^{2+}(0) \\
N^{2-}(0)
\end{array}\right)
$$

In the long run, under $N^{2+}(0)+N^{2-}(0)=1$, we have

$$
\begin{aligned}
& N^{2+}(t+2) /\left(\rho_{1} n\right)^{t / 2+1} \underset{t \rightarrow \infty}{\rightarrow} v_{11}\left[\left(w_{11}-w_{12}\right) N^{2+}(0)+w_{12}\right] \\
& N^{2-}(t+2) /\left(\rho_{1} n\right)^{t / 2+1} \underset{t \rightarrow \infty}{\rightarrow} v_{21}\left[\left(w_{11}-w_{12}\right) N^{2+}(0)+w_{12}\right]
\end{aligned}
$$

This establishes directly property a).

b) The dynamics of populations can be written

$$
\begin{aligned}
& N^{2+}(t+2)=\left(\rho_{1} n\right)^{t / 2+1} v_{11}\left[\left(w_{11}-w_{12}\right) N^{2+}(0)+w_{12}\right]+\left(\rho_{2} n\right)^{t / 2+1} v_{12}\left[\left(w_{21}-w_{22}\right) N^{2+}(0)+w_{22}\right] \\
& N^{2-}(t+2)=\left(\rho_{1} n\right)^{t / 2+1} v_{21}\left[\left(w_{11}-w_{12}\right) N^{2+}(0)+w_{12}\right]+\left(\rho_{2} n\right)^{t / 2+1} v_{22}\left[\left(w_{21}-w_{22}\right) N^{2+}(0)+w_{22}\right] \\
& N^{2+}(t+2)+N^{2-}(t+2)= \\
& \left(\rho_{1} n\right)^{t / 2+1}\left(v_{11}+v_{21}\right)\left[\left(w_{11}-w_{12}\right) N^{2+}(0)+w_{12}\right]+\left(\rho_{2} n\right)^{t / 2+1}\left(v_{12}+v_{22}\right)\left[\left(w_{21}-w_{22}\right) N^{2+}(0)+w_{22}\right]
\end{aligned}
$$

We know from Lemma $4 \mathrm{~d}$ that $w_{11}>w_{12}>0$, and $w_{21}<0<w_{22}$. Lemma $4 \mathrm{~b}$ established that $v_{11}, v_{21}, v_{22}>0, v_{12}<0$, and $v_{12}+v_{22}>0$ also hold.

Now notice that, if $N^{2+}(0)$ is decreased, then $N^{2+}(t+2)$ should go down. As $\rho_{1}>\rho_{2}$, $N^{2+}(t+2)+N^{2-}(t+2)$ drops too if $\left(v_{11}+v_{21}\right)\left(w_{11}-w_{12}\right)+\left(v_{12}+v_{22}\right)\left(w_{21}-w_{22}\right) \geq 0$. The expressions of matrices $V$ and $W$ given in Lemma 4 show that the left-hand side of this inequality is equal to 0 . However, we do not know if $N^{2-}(t+2)$ increases or decreases in the short run. Indeed, by the same reasoning as just before, this figure would go down if $v_{21}\left(w_{11}-w_{12}\right)+v_{22}\left(w_{21}-w_{22}\right) \geq 0$. Unfortunately this expression turns out to be equal to 
$-4\left(a_{12} \sqrt{\Delta}\right)$, which is negative. Therefore anything could happen in the short run as for the number of low human capital junior adults.

\section{Proof of Lemma 5}

We deduce from equation (15)

$\frac{d e_{+1}}{e_{+1}}=-\frac{d l}{h-l}$.

We deduce from equation (12)

$$
\begin{aligned}
& \left(\beta \frac{n B A^{\prime} /(A R)+B^{\prime}+B h}{B l+B^{\prime}}+2-\alpha-\beta\right) \frac{B d l}{B l+B^{\prime}}= \\
& \frac{(1-\alpha)(1-\beta)}{r\left(B l+B^{\prime}\right)^{1-\beta}} \frac{d B}{B}=\left[(1-\beta) \frac{n B A^{\prime} /(A R)+B^{\prime}+B h}{B l+B^{\prime}}-2+\alpha+\beta\right] \frac{d B}{B}<0
\end{aligned} .
$$

If we differentiate equation (8) and use the previous equation, we get equation (35). We deduce from equation (7)

$$
\frac{d \lambda\left(e_{+1}\right)}{\lambda\left(e_{+1}\right)}=(1-\alpha) \frac{A d e_{+1}}{A e_{+1}+A^{\prime}}=-(1-\alpha) \frac{B l+B^{\prime}}{B(h-l)+n B A^{\prime} /(A R)} \frac{B d l}{B l+B^{\prime}}
$$

If we substitute the above expression of $d l / l$ we get equation (36).

\section{Proof of Lemma 6}

Equation (5) and the conditions on the parameters imply that $r$ is positive and has an upper bound. Moreover, Lemma 1 established that $0<l<h$. The second expression of $E$ establishes

$$
0<\frac{(1-\alpha)^{2}}{n B A^{\prime} /(A R)+B^{\prime}+B h} \frac{1}{r} \frac{B^{\prime 2-\beta}}{B h+n B A^{\prime} /(A R)}<E<\frac{(1-\alpha)^{2}}{n B A^{\prime} /(A R)+B^{\prime}+B h} \frac{1}{r} \frac{\left(B h+B^{\prime}\right)^{2-\beta}}{n B A^{\prime} /(A R)}
$$

Each of the two factors appearing in the first expression of $E$ are smaller than 1.

\section{Proof of Proposition 2}

a) The change in the number of junior adults living in period 2, whose parents held a high level of human capital is, according to equation (20)

$$
\begin{aligned}
& d\left(a_{11}+a_{21}\right)=d\left\{\lambda\left(e_{+1}^{+}\right)\left[\pi\left(l^{+}\right)(1-c)+c\right]\right\}= \\
& \left\{\pi\left(l^{+}\right)(1-c)\left[\frac{d \lambda\left(e_{+1}^{+}\right)}{\lambda\left(e_{+1}^{+}\right)}+\frac{d \pi\left(l^{+}\right)}{\pi\left(l^{+}\right)}\right]+c \frac{d \lambda\left(e_{+1}^{+}\right)}{\lambda\left(e_{+1}^{+}\right)}\right\} \lambda\left(e_{+1}^{+}\right)
\end{aligned}
$$


We use equation (36) and get

$$
\begin{aligned}
& d\left(a_{11}+a_{21}\right)=\left\{\begin{array}{c}
\pi\left(l^{+}\right)(1-c)\left[1-\frac{\left[(1-\alpha)\left(B l^{+}+B^{\prime}\right)\right]^{2}}{\left[B h^{+}+B^{\prime}+n B A^{\prime} /(A R)\right]\left[B\left(h^{+}-l^{+}\right)+n B A^{\prime} /(A R)\right](1-\beta) r \pi\left(l^{+}\right)}\right] \\
-c \frac{\left[(1-\alpha)\left(B l^{+}+B^{\prime}\right)\right]^{2}}{\left[B h^{+}+B^{\prime}+n B A^{\prime} /(A R)\right]\left[B\left(h^{+}-l^{+}\right)+n B A^{\prime} /(A R)\right](1-\beta) r \pi\left(l^{+}\right)}
\end{array}\right\} \\
& \lambda\left(e_{+}^{+}\right) \frac{d \pi\left(l^{+}\right)}{\pi\left(l^{+}\right)}
\end{aligned}
$$

Equation (35) shows that $d\left(a_{11}+a_{21}\right)>0$ is equivalent to

$$
\begin{aligned}
& \pi\left(l^{+}\right)(1-c)\left[1-\frac{\left[(1-\alpha)\left(B l^{+}+B^{\prime}\right)\right]^{2}}{\left[B h^{+}+B^{\prime}+n B A^{\prime} /(A R)\right]\left[B\left(h^{+}-l^{+}\right)+n B A^{\prime} /(A R)\right](1-\beta) r \pi\left(l^{+}\right)}\right] \\
& <c \frac{\left[(1-\alpha)\left(B l^{+}+B^{\prime}\right)\right]^{2}}{\left[B h^{+}+B^{\prime}+n B A^{\prime} /(A R) \llbracket B\left(h^{+}-l^{+}\right)+n B A^{\prime} /(A R)\right](1-\beta) r \pi\left(l^{+}\right)}
\end{aligned}
$$

We use equation (14) and get

$$
\begin{aligned}
& \pi\left(l^{+}\right)(1-c)\left\{1-(1-\alpha) \frac{B l^{+}+B^{\prime}}{B h^{+}+B^{\prime}+n B A^{\prime} /(A R)}\left[1-\frac{1-\alpha}{1-\beta} \frac{B l^{+}+B^{\prime}}{B\left(h^{+}-l^{+}\right)+n B A^{\prime} /(A R)}\right]\right\} \\
& <c(1-\alpha) \frac{B l^{+}+B^{\prime}}{B h^{+}+B^{\prime}+n B A^{\prime} /(A R)}\left[1-\frac{1-\alpha}{1-\beta} \frac{B l^{+}+B^{\prime}}{B\left(h^{+}-l^{+}\right)+n B A^{\prime} /(A R)}\right] \\
& 1<\left[1+\frac{c}{1-c} \frac{1}{\pi\left(l^{+}\right)}\right](1-\alpha) \frac{B l^{+}+B^{\prime}}{B h^{+}+B^{\prime}+n B A^{\prime} /(A R)}\left[1-\frac{1-\alpha}{1-\beta} \frac{B l^{+}+B^{\prime}}{B\left(h^{+}-l^{+}\right)+n B A^{\prime} /(A R)}\right] \\
& =\left[1+\frac{c}{1-c} \frac{1}{\pi\left(l^{+}\right)}\right] E\left(h^{+}, l^{+}\right)
\end{aligned}
$$

Lemma 6 shows that $E\left(h^{+}, l^{+}\right)$has a positive lower bound. So, for $c$ near enough to 1 , the inequality is satisfied.

A similar computation shows that $d\left(a_{12}+a_{22}\right)>0$. Then, equation (33) establishes part a of the proposition.

b) We have

$$
d\left(a_{11}\right)=d\left\{\lambda\left(e_{+}^{+}\right)\left[\pi\left(l^{+}\right)(p-c q)+c q\right]\right\}
$$




$$
\left.\begin{array}{l}
d\left(a_{11}\right)=\left\{\begin{array}{c}
\pi\left(l^{+}\right)(p-c q)\left[1-\left[(1-\alpha)\left(B l^{+}+B^{\prime}\right)\right]^{2}\right. \\
-c q\left[B h^{+}+B^{\prime}+n B A^{\prime} /(A R)\right]\left[B\left(h^{+}-l^{+}\right)+n B A^{\prime} /(A R)\right](1-\beta) r \pi\left(l^{+}\right)
\end{array}\right] \\
{\left[(1-\alpha)\left(B l^{+}+B^{\prime}\right)\right]^{2}}
\end{array}\right\}
$$

Equation (35) shows that $d\left(a_{11}\right)<0$ is equivalent to

$$
\begin{aligned}
& \pi\left(l^{+}\right)(p-c q)\left[1^{1-}\left[\begin{array}{c}
{\left[(1-\alpha)\left(B l^{+}+B^{\prime}\right)\right]^{2}} \\
{\left[B h^{+}+B^{\prime}+n B A^{\prime} /(A R)\right]\left[B\left(h^{+}-l^{+}\right)+n B A^{\prime} /(A R)\right](1-\beta) r \pi\left(l^{+}\right)}
\end{array}\right]\right. \\
& >c q\left[\frac{\left[(1-\alpha)\left(B l^{+}+B^{\prime}\right)\right]^{2}}{\left[B h^{+}+B^{\prime}+n B A^{\prime} /(A R)\right]\left[B\left(h^{+}-l^{+}\right)+n B A^{\prime} /(A R)\right](1-\beta) r \pi\left(l^{+}\right)}\right.
\end{aligned}
$$

We use equation (14) and get

$$
\begin{aligned}
& 1>\left[1+\frac{c q}{p-c q} \frac{1}{\pi\left(l^{+}\right)}\right](1-\alpha) \frac{B l^{+}+B^{\prime}}{B h^{+}+B^{\prime}+n B A^{\prime} /(A R)}\left[1-\frac{1-\alpha}{1-\beta} \frac{B l^{+}+B^{\prime}}{B\left(h^{+}-l^{+}\right)+n B A^{\prime} /(A R)}\right] \\
& =\left[1+\frac{c q}{p-c q} \frac{1}{\pi\left(l^{+}\right)}\right] E\left(h^{+}, l^{+}\right)
\end{aligned}
$$

According to lemma 6, a sufficient condition for this inequality to hold is $1+\frac{c q}{p-c q} \frac{1}{\pi\left(l^{+}\right)}<\frac{1}{\bar{E}}$, with $1 / \bar{E}>1$

For $q$ near enough to 0 , the inequality is satisfied. A similar computation shows that $d\left(a_{12}\right)<0$. Then, equation (31) establishes part $\mathrm{b}$ of the proposition. 
Figure 1

Figure 2. Estimated and projected population size with and without AIDS, 38 African countries, $1995-2000$ to $2020-2025$

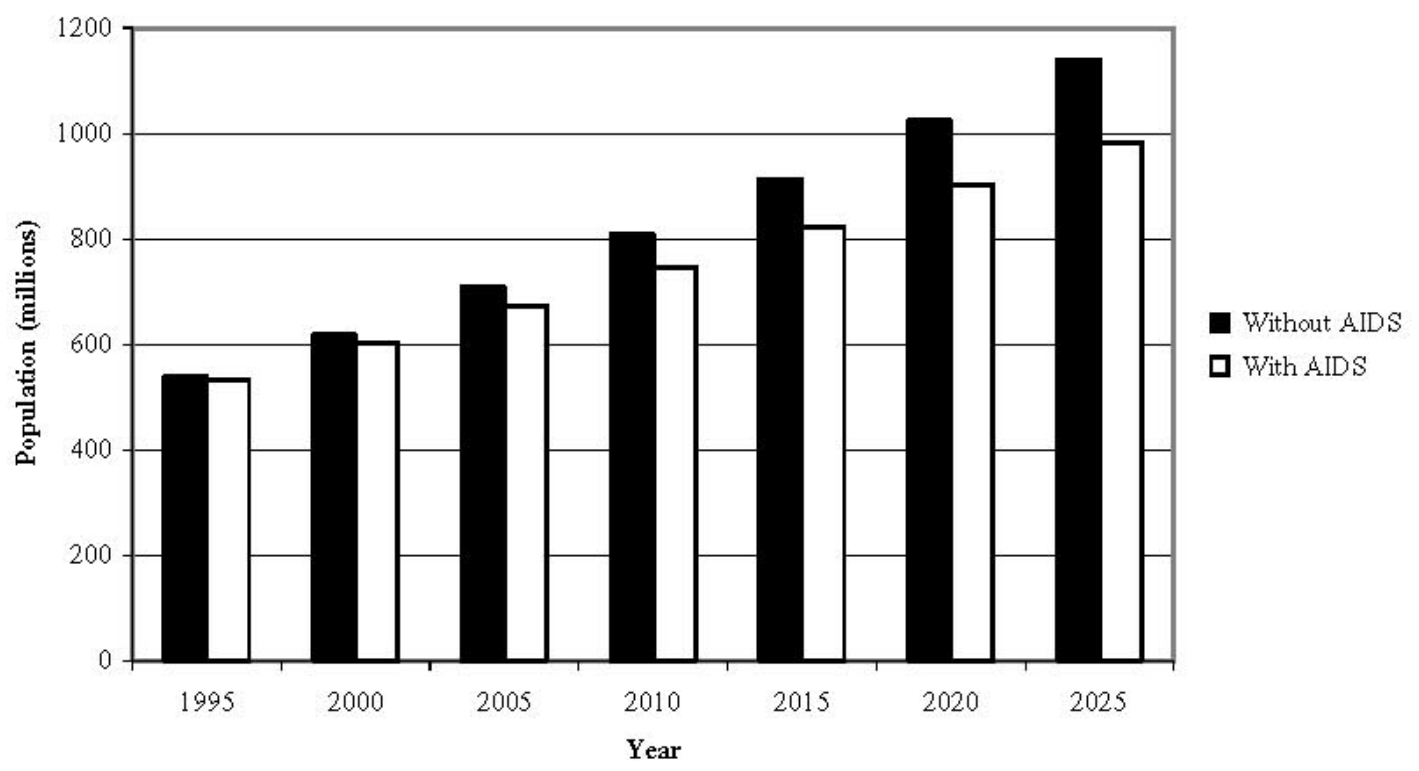

Source: World Population Prospects: The 2002 Revision, CD-ROM (United Nations, Department of Economic and Social Affairs, Population Division publication, Sales No. E.03.XIII.8). 
Figure 2

\section{Child Mortality Rate* With and Without \\ AIDS, Southern Africa: 1996}

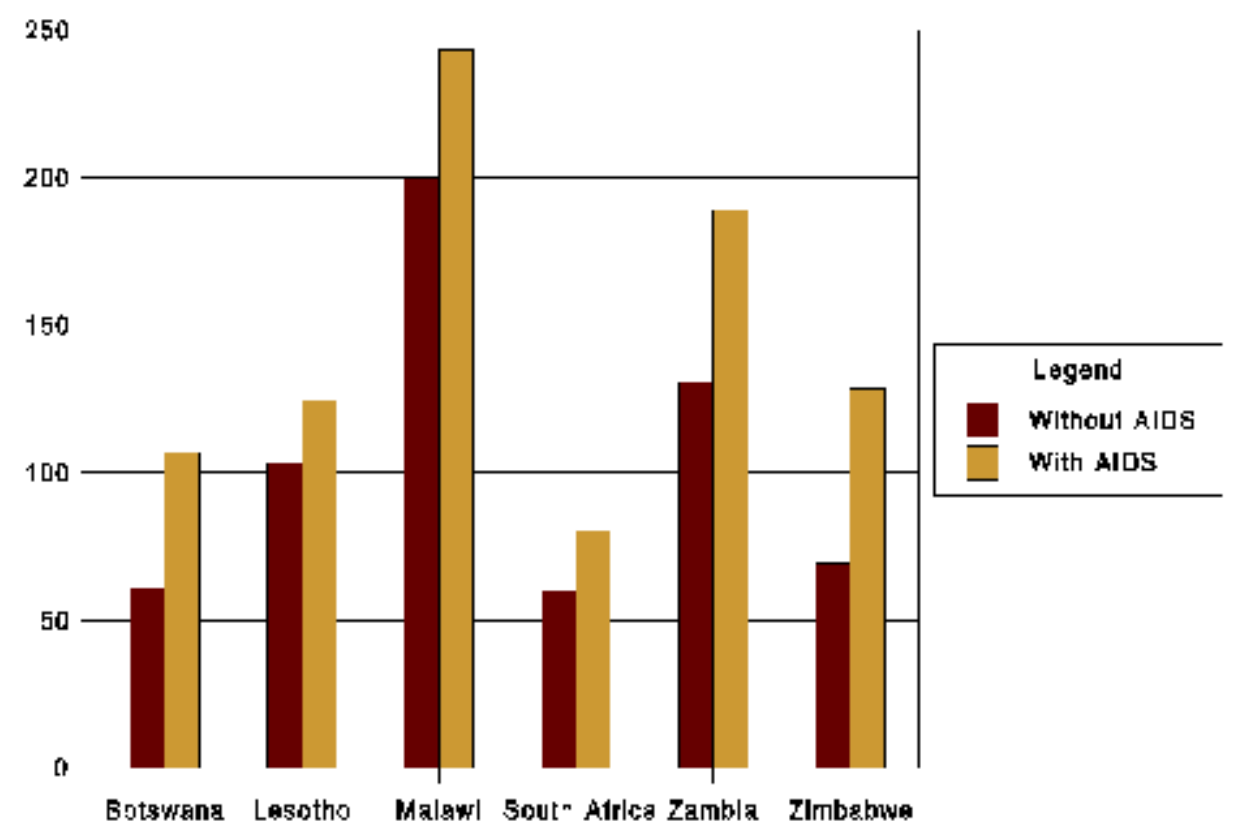

Child Mortality Rate* With and Without

AIDS, Southern Africa: 2010

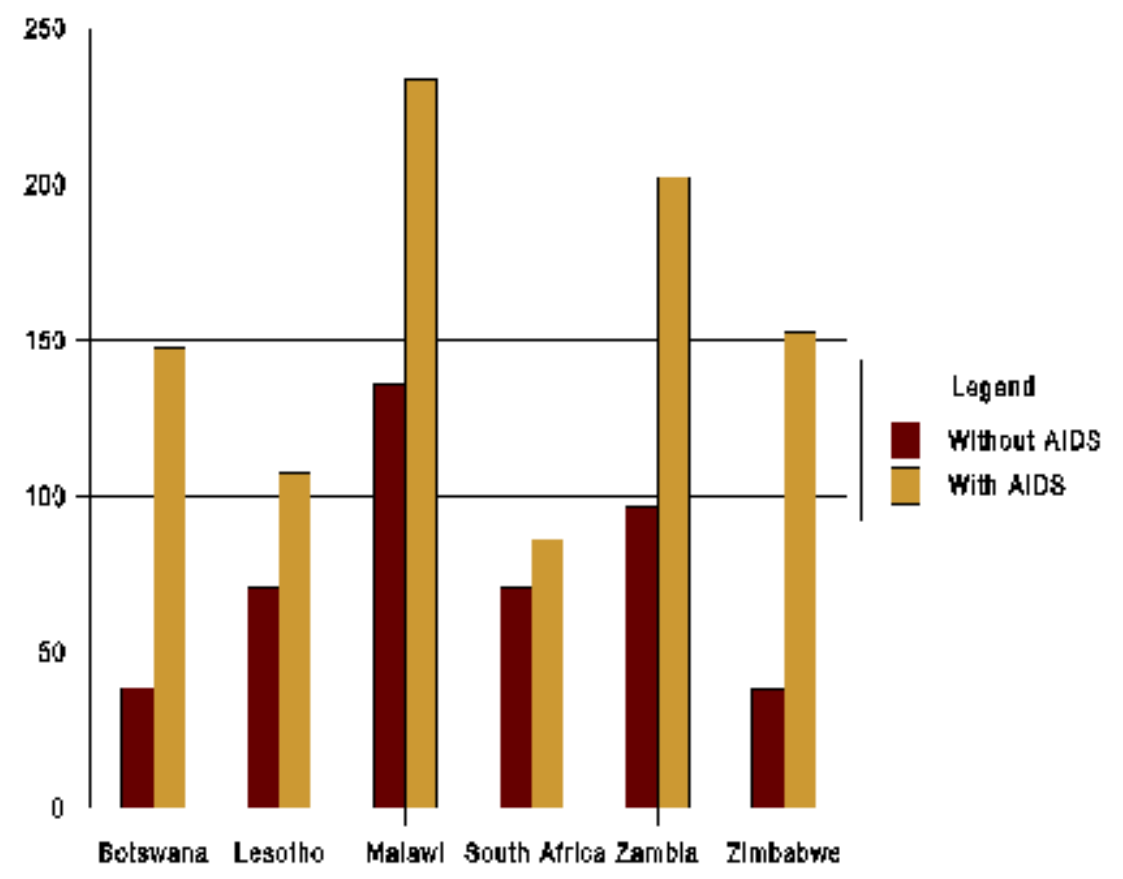

*Child mortality rate is the number of children dying before age 5 per 1,000 live births. Source: International Programs Center - Population Division US Bureau of the Census, Washington, DC 


\section{Figure3}

Figure 4. Population size with and without AIDS, Botswana, 2000 and 2025

2000
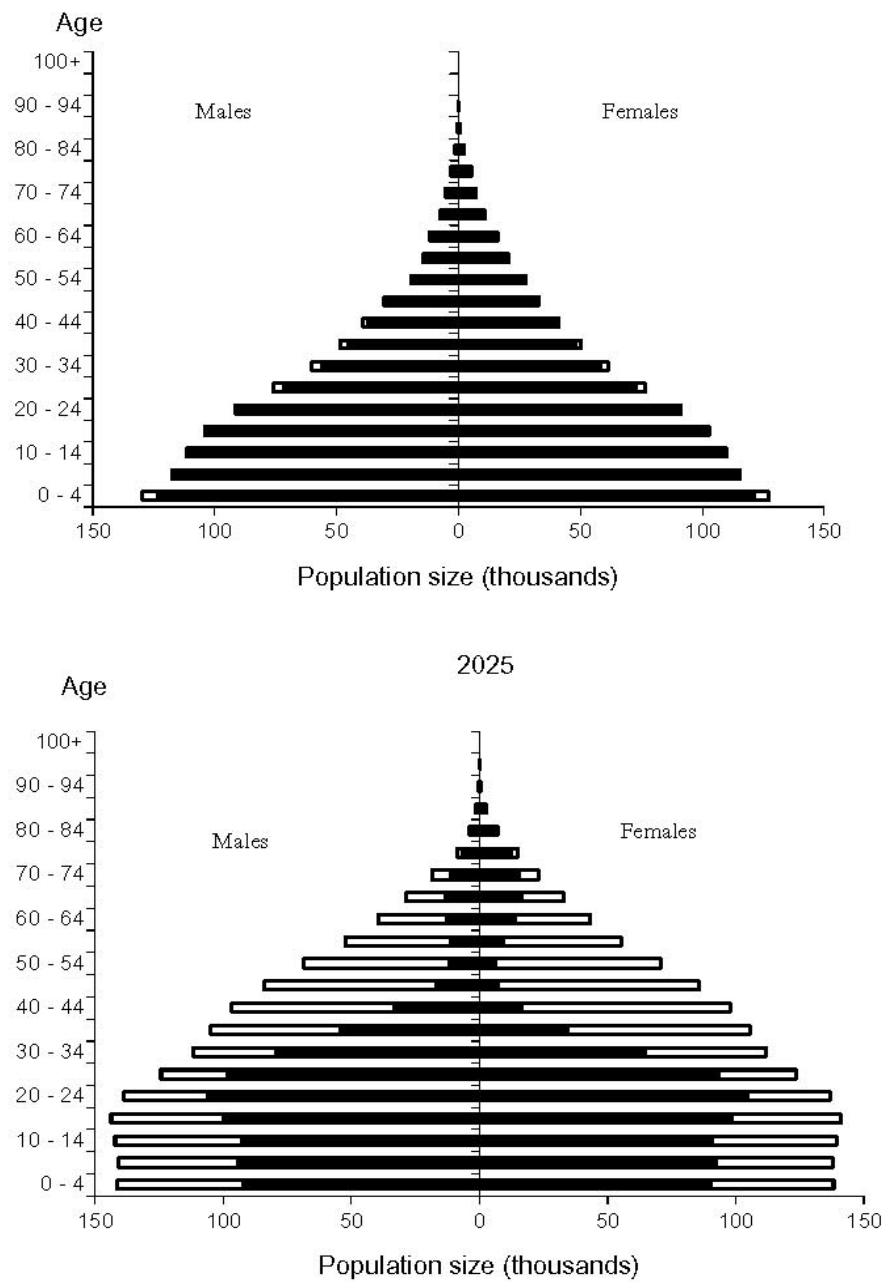

Source: World Population Prospects:The 2002 Revision, CD-ROM (United Nations, Department of Economic and Social Affairs, Population Division publication, Sales No. E.03.XIII.8)

NOTE: Unshaded bars represent the hypothetical size of the population in the absence of AIDS. Shaded bars represent the actual estimated and projected population 